${ }^{1}$ UCL Cancer Institute, University College London, London, UK.

${ }^{2}$ Division of Surgery and Interventional Science,

University College London, London, UK.

${ }^{3}$ Department of ENT, Barts Health NHS Trust, London, UK.

${ }^{4}$ Department of ENT, Cambridge University Hospitals NHS Trust, Cambridge, UK

${ }^{5}$ School of Biosciences, University of Kent, Canterbury, UK.

${ }^{6}$ School of Cancer Sciences, Cancer Research UK Centre Faculty of Medicine, University of Southampton, Southampton, UK.

凶e-mail:m.lechner@ucl.ac.uk. t.fenton@soton.ac.uk https://doi.org/10.1038 s41571-022-00603-7

\title{
HPV-associated oropharyngeal cancer: epidemiology, molecular biology and clinical management
}

Matt Lechner $\mathbb{B}^{1,2,3 凶}$, Jacklyn Liu ${ }^{1}$, Liam Masterson ${ }^{4}$ and Tim R. Fenton $\mathbb{1}^{5,6 \times}$

Abstract | Human papillomavirus (HPV)-positive $\left(\mathrm{HPV}^{+}\right)$oropharyngeal squamous cell carcinoma (OPSCC) has one of the most rapidly increasing incidences of any cancer in high-income countries. The most recent (8th) edition of the UICC/AJCC staging system separates HPV ${ }^{+}$OPSCC from its $\mathrm{HPV}^{-n e g a t i v e ~}\left(\mathrm{HPV}^{-}\right)$counterpart to account for the improved prognosis seen in the former. Indeed, owing to its improved prognosis and greater prevalence in younger individuals, numerous ongoing trials are examining the potential for treatment de-intensification as a means to improve quality of life while maintaining acceptable survival outcomes. In addition, owing to the distinct biology of $\mathrm{HPV}^{+}$OPSCCs, targeted therapies and immunotherapies have become an area of particular interest. Importantly, OPSCC is often detected at an advanced stage owing to a lack of symptoms in the early stages; therefore, a need exists to identify and validate possible diagnostic biomarkers to aid in earlier detection. In this Review, we provide a summary of the epidemiology, molecular biology and clinical management of $\mathrm{HPV}^{+}$OPSCC in an effort to highlight important advances in the field. Ultimately, a need exists for improved understanding of the molecular basis and clinical course of this disease to guide efforts towards early detection and precision care, and to improve patient outcomes.

Oropharyngeal squamous cell carcinoma (OPSCC) comprises cancers of the tonsils, base of tongue, soft palate and uvula (FIG. 1). Like other head and neck squamous cell carcinomas (HNSCCs), OPSCC has historically been linked to alcohol and tobacco consumption. A reduction in the prevalence of smoking in most high-income countries over the past 20 years has led to a decline in the incidence of HNSCC; however, carcinogenic human papillomavirus (HPV) infection has emerged as an important risk factor that has driven an increase in the incidence of OPSCC over the same period. More specifically, HPV now accounts for $71 \%$ and $51.8 \%$ of all OPSCCs in the USA and UK, respectively ${ }^{1-4}$. Of these, $85-96 \%$ are caused by HPV-16 infections and are therefore expected to be preventable by prophylactic HPV vaccination, which is known to be effective in preventing HPV-associated cervical neoplasia and is now being administered to both boys and girls in several countries 4,5 . The most recent edition of the American Joint Committee on Cancer (AJCC) staging system defined HPV-positive $\left(\mathrm{HPV}^{+}\right)$ and $\mathrm{HPV}$-negative $\left(\mathrm{HPV}^{-}\right)$OPSCCs as separate entities, with distinct molecular profiles, tumour characteristics and outcomes ${ }^{6}$ (TABLE 1). Importantly, the former is associated with a more favourable prognosis ${ }^{7}$. In this Review, we provide a comprehensive overview of $\mathrm{HPV}^{+} \mathrm{OPSCC}$, focusing on how our increasing knowledge of disease biology has informed clinical practice and is guiding the pursuit of improved treatments.

\section{Epidemiology \\ Rising incidence, particularly in men}

Among all cancers, OPSCC has one of the most rapidly rising incidences in high-income countries ${ }^{8,9}$. An increasing incidence of this disease has been observed in the UK, USA, across Europe, New Zealand and in parts of Asia ${ }^{9-19}$. In both the UK and the USA, the incidence of oropharyngeal cancer in men has surpassed that of cervical cancer in women ${ }^{8}$ (FIG. 1). Globally, the percentage of OPSCCs that are $\mathrm{HPV}^{+}$was reported in 2021 to be $33 \%$; however, prevalence varies considerably depending on the geographical region, with estimates ranging from $0 \%$ in southern India to $85 \%$ in Lebanon ${ }^{20}$.

$\mathrm{HPV}^{+}$OPSCC is more prevalent than $\mathrm{HPV}^{-}$OPSCC among those who do not consume tobacco or alcohol; however, a substantial history of tobacco and alcohol use remains prominent in patients with the former and is associated with worse outcomes ${ }^{21,22}$. Furthermore, sexual behaviour is an established risk factor for $\mathrm{HPV}^{+}$OPSCC, with a strong association observed between number of 


\section{Key points}

- The incidence of human papillomavirus-associated oropharyngeal cancer (HPV OPSCC) is expected to continue to rise over the coming decades until the benefits of gender-neutral prophylactic HPV vaccination begin to become manifest.

- The incidence of $\mathrm{HPV}^{+} \mathrm{OPSCC}$ appears to be highest in high-income countries, although more epidemiological data are needed from low- and middle-income countries, in which HPV vaccination coverage remains low.

- The substantially better prognosis of patients with $\mathrm{HPV}^{+}$OPSCC compared to those with HPV-OPSCC has been recognized in the American Joint Committee on Cancer TNM8 staging guidelines, which recommend stratification by HPV status to improve staging.

- The molecular biology and genomic features of $\mathrm{HPV}^{+}$OPSCC are similar to those of other HPV-associated malignancies, with HPV oncogenes (E6 and E7) acting as key drivers of pathogenesis.

- Treatment de-intensification is being pursued in clinical trials, although identifying the $\sim 15 \%$ of patients with $\mathrm{HPV}^{+}$OPSCC who have recurrent disease, and who therefore require more intensive treatment, remains a key challenge.

lifetime oral sex partners and incidence of the disease ${ }^{2,23}$. As mentioned above, this association might partially reflect an observed gender disparity given that men are more likely than women to report increased numbers of sexual partners ${ }^{24}$. Risk of oral HPV infection is associated with an increased number of recent (within the past 3 months) oral and vaginal sex partners ${ }^{24}$.

The incidence of both $\mathrm{HPV}^{+}$and HPV- OPSCC has increased over the past two decades,although evidence suggests that the former is increasing more rapidly. In Denmark, a threefold increase in $\mathrm{HPV}^{+} \mathrm{OPSCC}$ was observed between 2000 and 2017, compared with a twofold increase for $\mathrm{HPV}^{-}$disease ${ }^{13}$. Comparatively, a more rapid increase in $\mathrm{HPV}^{+} \mathrm{HNSCC}$, particularly tonsillar squamous cell carcinoma (SCC), has been observed in Taiwan, compared with HPV ${ }^{-}$HNSCC $^{11}$. In Italy, the incidence of $\mathrm{HPV}^{+}$OPSCCs (as a percentage of all OPSCCs) increased from $16.7 \%$ in $2000-2006$ to $46.1 \%$ in 2013-2018 (REF. ${ }^{14}$ ). Lower-to-middle income countries in southern Asia and sub-Saharan Africa bear the vast majority of the global burden of HPV-associated cervical cancer, although epidemiological reports that focus on $\mathrm{HPV}^{+} \mathrm{OPSCC}$ are scarce and, as a result, whether similar rising trends are absent or simply thus far undetected in these regions remains unclear ${ }^{25}$.

From the few reports available, the prevalence of $\mathrm{HPV}^{+}$OPSCCs in sub-Saharan Africa is low, with very few cases reported to date despite high rates of HPV-associated cervical cancer ${ }^{26-30}$. In an investigation of $\mathrm{HPV}^{+} \mathrm{OPSCC}$ in Mozambique, the authors proposed that the low prevalence of $\mathrm{HPV}^{+}$OPSCCs in their cohort (14.5\%) might reflect the limited practice of oral sex in the region ${ }^{26}$. This observation has been reiterated by other investigators, who observed low rates of oral HPV infection among HIV-infected individuals in northwest Cameroon and attribute this, at least in part, to limited oral sexual behaviours, relative to higher-income countries ${ }^{27}$.

Historically, most $\mathrm{HPV}^{+}$OPSCCs occur in men, which might reflect differences in both susceptibility and infection transmissibility through sexual activities, although this has yet to be fully elucidated ${ }^{4,31-33}$. However, an increase in incidence has been observed among white women in the USA ${ }^{32}$. In a recent meta-analysis including data from 12 studies, the authors observed a similar prevalence of HPV-driven OPSCCs in both males and females, despite most of the assessed patients with OPSCC being male ${ }^{34}$.

The prevalence of $\mathrm{HPV}^{+}$OPSCC was previously reported to decrease with increasing age; however, the burden of disease has begun to shift towards older men as a result of a birth cohort effect ${ }^{4,33}$. In one study, the median age at diagnosis increased from 53 to 58 years of age between 1998 and $2013\left(\right.$ REF $^{35}$ ) while another study reported a similar increase, from 52 to 59 years of age between 2002 and 2017 (REF. ${ }^{36}$ ). A rapidly increasing incidence in white men $\geq 65$ years of age has also been observed, and nearly $10 \%$ of cases have been reported in those $\geq 70$ years of age ${ }^{32,36}$. Nevertheless, increased rates of $\mathrm{HPV}^{+}$OPSCC continue to be evident in both younger and older adults and, although the burden is shifting towards older adults, most cases remain in those $<65$ years of age $e^{31,37,38}$.

In the USA, a higher prevalence of $\mathrm{HPV}^{+} \mathrm{OPSCC}$ has been observed in white individuals than in those of other ethnicities ${ }^{39,40}$. In an analysis of the Surveillance, Epidemiology and End Results (SEER) database, a significant increase in rates of oropharyngeal cancer in men of white or Hispanic ethnicity, and in men of other ethnicities was observed, albeit with a decrease in Black $m^{32}$. However, another report describes a significantly more rapid increase in the prevalence of $\mathrm{HPV}^{+} \mathrm{OPSCCs}$ in Black and Hispanic American individuals compared with white American individuals ${ }^{9,32}$. These results might reflect a greater decrease in $\mathrm{HPV}^{-}$disease in Black men compared with white and Hispanic men, resulting in the observed relative increase in $\mathrm{HPV}^{+}$disease; however, this suggestion has yet to be confirmed. In parallel with the increased incidence in white men in the USA, higher socioeconomic status is also associated with an increased incidence of $\mathrm{HPV}^{+} \mathrm{OPSCC}^{40}$.

Importantly, most studies of the epidemiology of $\mathrm{HPV}^{+}$OPSCC have been conducted in the USA and are therefore not necessarily generalizable to other parts of the world, where differences in culture and behaviour might influence the various lifestyle factors that have a role in the aetiology of $\mathrm{HPV}^{+}$ OPSCC. As such, further studies in geographically diverse, and particularly non-Western regions, are needed to inform region-specific guidelines, particularly with regard to clinical management and targeted public health measures.

\section{The role of HPV vaccination}

A need remains for improved cancer prevention that is responsive to ever-changing societal norms. No screening methods enabling the earlier detection of OPSCC are currently available; therefore, widespread prevention can be achieved only through large-scale vaccination programmes. HPV vaccination has been offered to girls for nearly two decades and has led to decreased rates of cervical cancer in high-income countries, where HPV vaccination is widely available ${ }^{5,41}$ One might argue that the herd immunity established through widespread vaccination of girls might preclude the need for vaccination 
of boys, considering the costs associated with such mass vaccination programmes. However, the universal vaccination of girls will probably not completely mitigate the risks of HPV infection and consequent development of HPV-related cancers in men ${ }^{42}$. Indeed, such a policy must take into account the population of men who have sex with men, as well as those who have sexual partners originating from geographical regions that lack a comprehensive HPV vaccination programme, even in girls. Furthermore, variability in vaccination uptake owing to practical, societal and cultural barriers will probably continue to hinder the ability of populations to achieve the levels of immunity that are necessary to prevent future HPV-related malignancies.

Several countries have now extended nationwide vaccination programmes to boys, including Australia, Austria, Germany, Italy, New Zealand, the UK and the USA. Australia was one of the first countries to implement a gender-neutral programme and has demonstrated high levels of vaccine uptake, with $75.9 \%$ and $80.2 \%$ of boys and girls, respectively, completing a three-dose regimen ${ }^{43}$. By comparison, half of

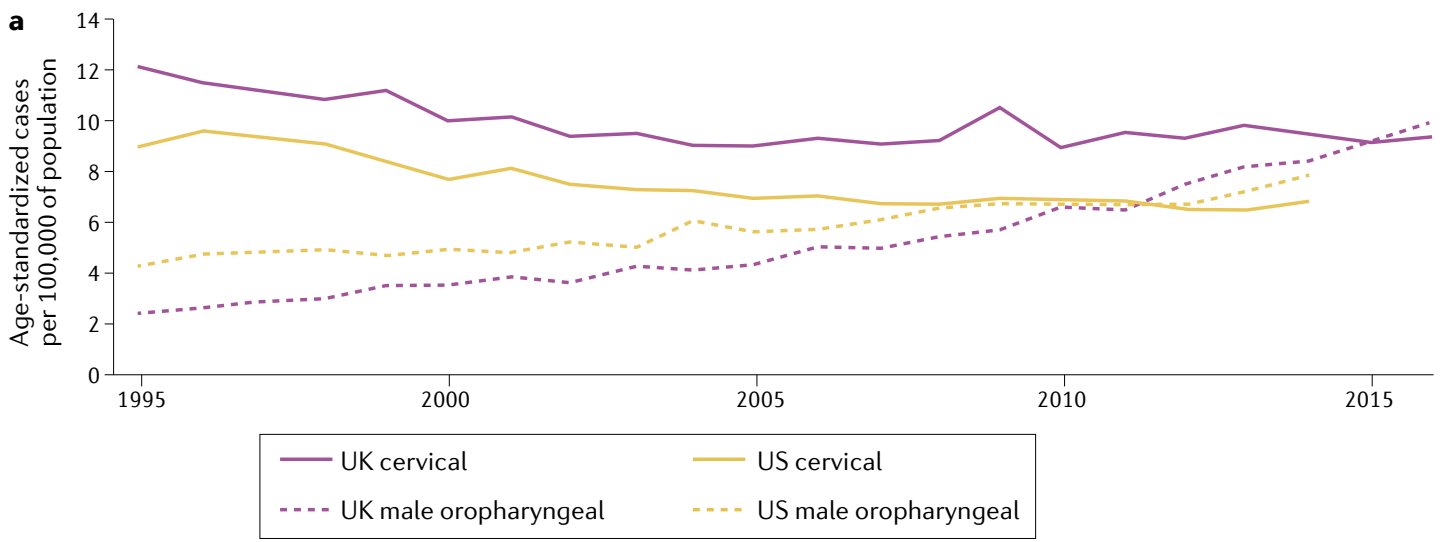

b
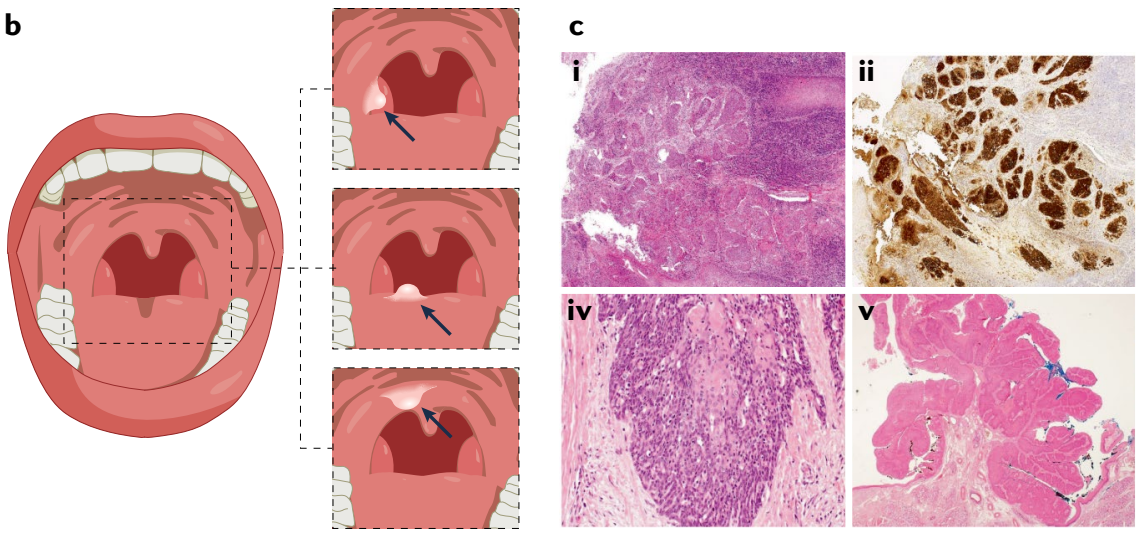

iii
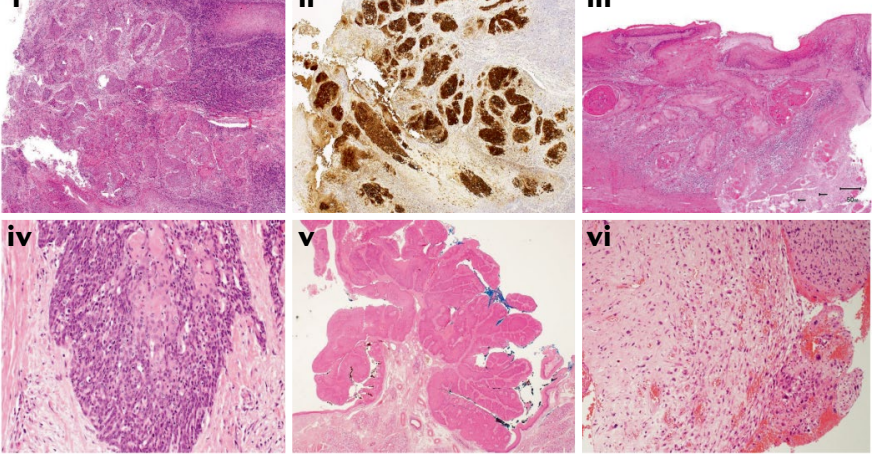

Fig. 1 Incidence, anatomical locations and histological appearance of $\mathrm{HPV}^{+}$oropharyngeal cancers. a | Directly age-standardized rates per 100,000 population of newly diagnosed cases of cervical and oropharyngeal cancer in the UK and the USA. For male oropharyngeal cancers (pink dotted line) and cervical cancers (pink solid line) in the UK from 1995 to 2016, data are sourced from the UK Office for National Statistics cancer data ${ }^{241}$. Male oropharyngeal cancers include those of the base of tongue, uvula, tonsil and oropharynx, stratified for different types of squamous cell carcinoma (SCC) (as for the US data). Observed age-standardized rates per 100,000 population of newly diagnosed cases of cancer; for oropharyngeal cancers among men (yellow dotted line) and cervical cancers (yellow solid line) from 1995 to 2014 obtained from registries within the Surveillance, Epidemiology, and End Results (SEER) programme ${ }^{242}$. Oropharyngeal cancers included those of the base of tongue, lingual tonsil, soft palate not otherwise specified, uvula, tonsil, oropharynx and Waldeyer's ring. Cervical cancers include all histological subtypes. b | Basic anatomy of the oropharynx, with inset images from top to bottom depicting human papillomavirus-positive oropharyngeal squamous cell carcinoma (HPV+ $\mathrm{OPSCC}$ ) located at the base of tongue (the anterior two-thirds), the soft palate and the tonsil. c| Histological appearance, clockwise from top left. i | Non-keratinizing SCC. ii | Non-keratinizing SCC with immunohistochemical staining for p16; morphology is monomorphic, ovoid, hyperchromatic with inconspicuous cytoplasm. This sample also features increased mitosis, apoptosis and comedo-type necrosis. iii | Keratinizing SCC, featuring filiform projections, a thickened, nonmalignantappearing stratified squamous epithelium, hyperparakeratosis and keratin plugging. iv | Basaloid SCC featuring variable foci of squamous differentiation. $\mathbf{v}$ | Papillary SCC with early invasion, featuring predominant filiform processes with minimal or absent keratinization, frequent mitosis and full-thickness dysplasia with a basaloid cell morphology. vi $\mid$ Spindlecell carcinoma, featuring a biphasic tumour composed of SCC and malignant spindle-cell component, exhibits polypoid growth. All images in c shown at $40 \times$ magnification. 
Table 1 | Comparison of the key characteristics of $\mathrm{HPV}^{+}$and $\mathrm{HPV}^{-}$OPSCCs

\begin{tabular}{|c|c|c|}
\hline Characteristics & $\mathrm{HPV}^{+}$OPSCC & HPV- OPSCC \\
\hline \multicolumn{3}{|l|}{ Patient characteristics } \\
\hline $\begin{array}{l}\text { Average age at diagnosis } \\
\text { (years) }\end{array}$ & $59^{a}$ & $60(P<0.001)^{38}$ \\
\hline Sex & $86.9 \%$ male & $76.8 \%$ male $(P<0.001)^{38}$ \\
\hline Ethnicity & $90 \%$ white & $75.9 \%$ white $(P<0.001)^{38}$ \\
\hline Role of smoking & \multicolumn{2}{|c|}{ Rising incidence of HPV+ OPSCC in smokers, as well as in nonsmokers ${ }^{38}$} \\
\hline Role of alcohol & \multicolumn{2}{|l|}{ HPV $^{-}$OPSCC associated with greater alcohol consumption ${ }^{7}$} \\
\hline Role of sexual history & \multicolumn{2}{|c|}{ High number of sexual partners a risk factor for $\mathrm{HPV}^{+} \mathrm{OPSCC}^{7}$} \\
\hline \multicolumn{3}{|l|}{ Tumour characteristics } \\
\hline Incidence per 100,000 & 4.62 & $1.82\left(\mathrm{REF}^{38}\right)$ \\
\hline Anatomical location & $\begin{array}{l}\text { More prevalent in oropharynx ( } 94.2 \% \text { HNSCC); } \\
\text { specifically the base of tongue and tonsils }\end{array}$ & $\begin{array}{l}\text { Less prevalent in the oropharynx } \\
(72.8 \% \text { HNSCC })^{38}\end{array}$ \\
\hline Stage (AJCC 7th edn) & $\begin{array}{l}\text { Early stage }(\mathrm{T} 1-2) \text {; frequently with nodal metastasis at } \\
\text { presentation }{ }^{156}\end{array}$ & All stages $(\mathrm{T} 1-4)^{38}$ \\
\hline $\begin{array}{l}\text { Histopathological } \\
\text { appearance }\end{array}$ & Immature, basal-like/basaloid, non-keratinizing ${ }^{156}$ & Frequently keratinizing SCC \\
\hline Cancer-specific mortality & \multicolumn{2}{|c|}{$\mathrm{HPV}^{+} \mathrm{OPSCC}$ associated with a more favourable prognosis $(\mathrm{aHR} 0.40, \mathrm{P}<0.001)^{38}$} \\
\hline \multicolumn{3}{|l|}{ Biological characteristics } \\
\hline Genetic alterations & $\begin{array}{l}\text { More frequent alterations in genes encoding DNA } \\
\text { damage response proteins, FGF and JAK-STAT } \\
\text { signalling proteins, as well as immune-related genes } \\
\text { such as HLA-A/B; PIK3CA mutations more commonly } \\
\text { observed }^{95}\end{array}$ & $\begin{array}{l}\text { Aberration of TP53 and cell-cycle } \\
\text { pathways (such as CDKN2A loss); } \\
\text { oxidative stress regulation more } \\
\text { frequently mutated }{ }^{95}\end{array}$ \\
\hline Other aberrations & p53 and Rb degradation by E6 and E7, respectively 243 & NR \\
\hline
\end{tabular}

all adolescents in the USA in 2018 had completed the recommended three-dose regimen and nearly one-third were unvaccinated ${ }^{44}$. In the UK, school-based vaccination was extended to include boys in September 2019. In the subsequent academic year, the first dose of a two-dose vaccination regimen was given to $59.2 \%$ and $54.4 \%$, respectively, of girls and boys ${ }^{45}$. Importantly, owing to school closures as a result of the ongoing COVID-19 pandemic, roll-out of the programme was interrupted. Therefore, the true uptake from this first year of a gender-neutral vaccination programme in the UK has yet to be determined.

Several barriers to vaccination persist, including parental concerns over vaccine safety, socioeconomic factors and an overall lack of awareness ${ }^{8,46-48}$. In a survey of 725 adults between 27 and 45 years of age in the USA, only $36 \%$ of responders were aware that HPV infection can cause noncervical cancers ${ }^{49}$. In a separate survey of roughly 1,000 UK parents with children in school years 5-7 (aged 9-12 years), before the extension of vaccination to boys in 2019, only half had heard of HPV and fewer than 25\% knew that HPV vaccination would be offered to boys ${ }^{50}$. In this study, appropriate education of parents led to approximately two-thirds indicating that they would allow their child to be vaccinated, while only $10 \%$ would not. This finding implies that the provision of appropriate information to parents by health-care providers and public health administrators can lead to a high level of vaccine acceptance. Further education can help to assuage additional concerns for those who are undecided and demonstrate so-called flexible hesitancy ${ }^{50}$. Importantly, improved knowledge on the part of health-care providers is also needed to effectively implement large-scale vaccination programmes. In results from a survey of health-care professionals in the UK published in 2020, more than one-third of participants indicated the need for improved training, with $76 \%$ reporting that they felt adequately informed ${ }^{51}$. In a survey of general practitioners in the UK, $74 \%$ recognized HPV infection as a risk factor for OPSCC, although fewer than half were aware that being male was a risk factor for $\mathrm{HPV}^{+} \mathrm{OPSCC}^{52}$.

With regard to the efficacy of vaccination in preventing OPSCC, data from a registry report published in 2021 demonstrate a substantially higher risk of OPSCC in those who are not vaccinated compared with those who are ${ }^{53}$. Importantly, such conclusions might be premature as the effects of herd immunity owing to vaccination of females are an important confounder, and the true effects of gender-neutral vaccination are still emerging. Nevertheless, this finding is encouraging and reflects the efficacy of vaccination against OPSCC, which is supported by several reports that indicate effectiveness against HPV infection. For example, in a prospective cohort of $>7,000$ young women in Costa Rica, a 93.3\% decrease in oral HPV-16/18 infections was observed in those vaccinated against HPV relative to those vaccinated against hepatitis $\mathrm{A}^{54}$. In a subsequent 


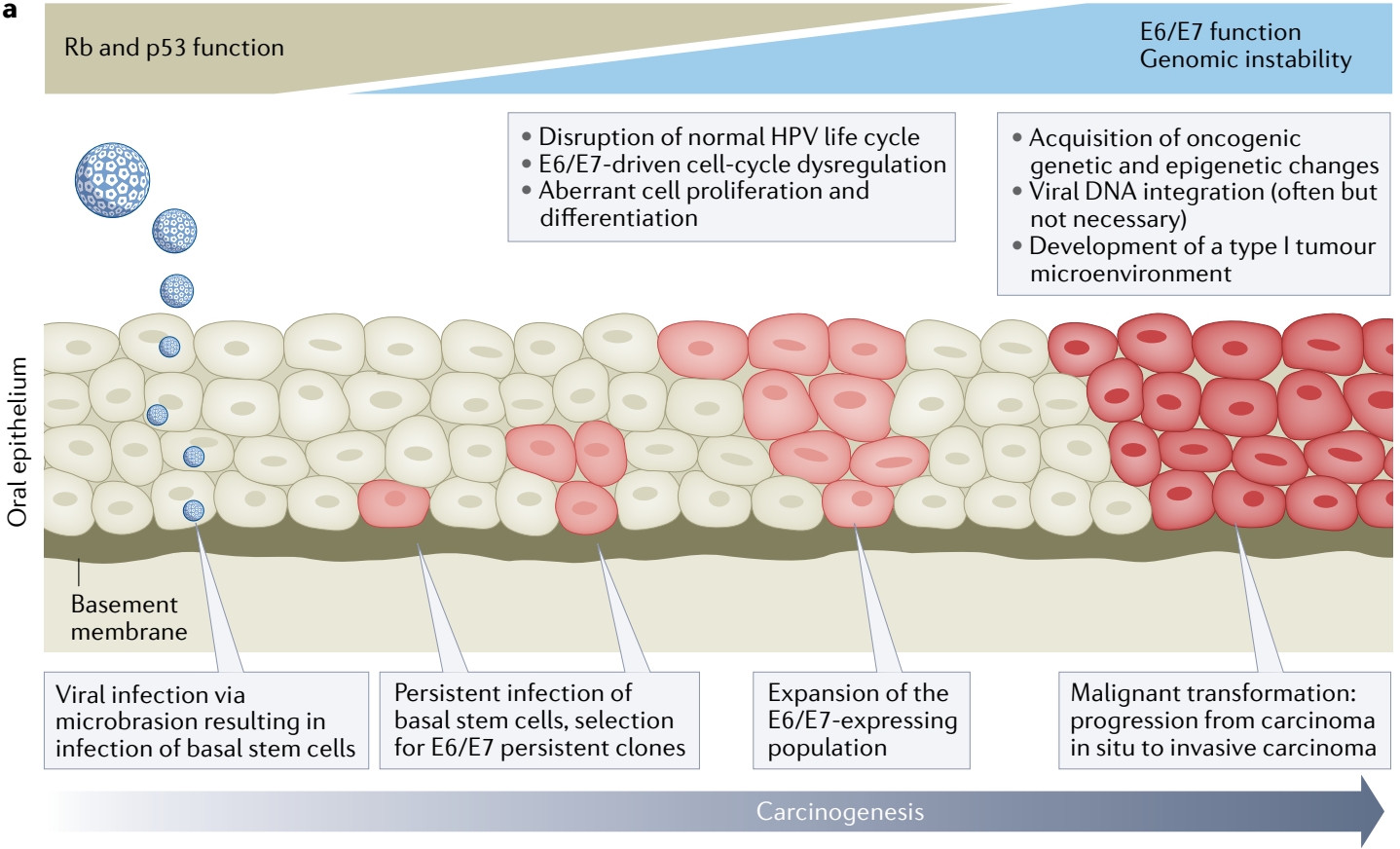

b

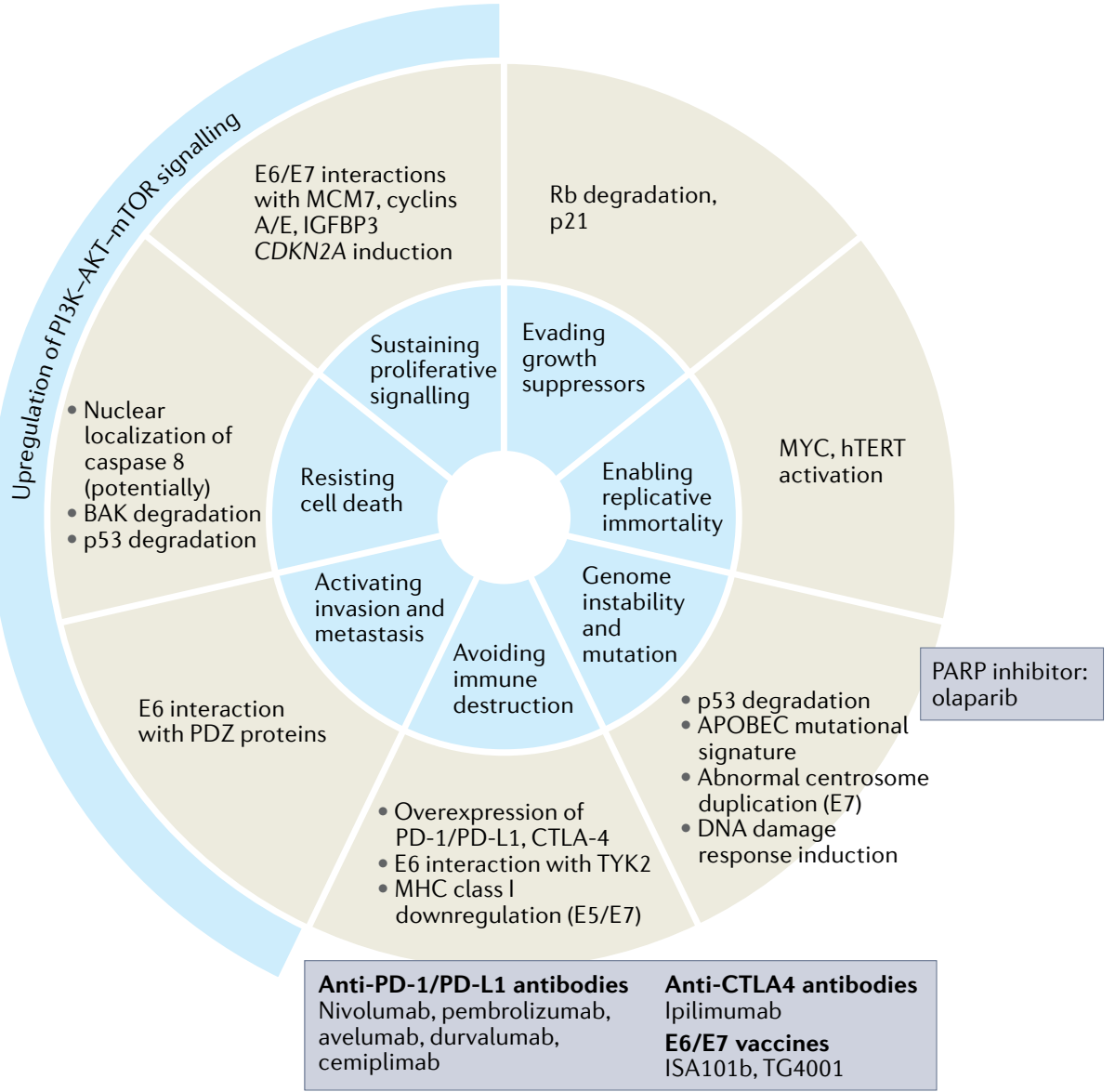

analysis of survey data from 2,627 adults in the USA (18-33 years of age between 2011 and 2014), the prevalence of oral HPV-6/11/16/18 infection was significantly lower in vaccinated men compared with unvaccinated men $(0.0 \% \text { versus } 2.13 \% \text {; adjusted } P=0.007)^{55}$.
Despite the introduction of HPV vaccination programmes for boys in several countries in the past few years and demonstrable efficacy against oral HPV infection, $\mathrm{HPV}^{+} \mathrm{OPSCC}$ rates are likely to rise further over the next 20-30 years, before the full benefits of 
4 Fig. 2 | Oncogenesis of $\mathrm{HPV}^{+}$oropharyngeal cancers. a| Major events in the development of human papillomavirus (HPV)-driven malignancy based on the established stepwise model of cervical carcinogenesis. HPV infection is established in the basal layer of the epithelium, with access facilitated either through micro-abrasions or, in the case of the oropharynx, potentially owing to the reticulated nature of the epithelium of the tonsillar crypts. Productive infections are usually cleared by the immune system but if not, deregulation of $E 6$ and $E 7$ expression can occur, causing exit from the productive viral life cycle and the development of neoplasia (in the cervix this is evident as lesions detectable by screening but no such lesions have been identified in the oropharynx). E6 and E7 suppress important mechanisms of tumour suppression and cause epigenetic changes, which can combine with somatic alterations in the host cell genome to cause transformation and progression to malignancy. In the cervix, these events typically occur over the course of 10-20 years after the causative HPV infection ${ }^{60,61}$. b $\mid$ Schematic showing how HPV-driven oncogenic processes act to enable seven of the eight hallmarks of cancer originally defined by Hanahan and Weinberg ${ }^{68}$ and how experimental therapies are able to disable one or more of these hallmarks ${ }^{66,67,240}$. carcinogenesis in the cervix have been studied extensively (FIC. 2). $\mathrm{HPV}^{+}$carcinogenesis is largely driven by two viral early genes (E6 and $E 7$, often referred to as HPV oncogenes), the physiological function of which is to trigger cell-cycle entry in the basal layer of the epithelium and thus permit viral genome replication. Increased expression of $E 6$ and $E 7$ is often associated with integration of hrHPV DNA into the host genome, although carcinogenesis can occur in the absence of integration: whole-genome sequencing of $103 \mathrm{HPV}^{+}$ OPSCCs revealed evidence of viral integration in $74 \%$ of patients, with the remaining tumours harbouring episomal $\mathrm{HPV}^{64}$, a similar frequency of integration to that seen in HPV- $16^{+}$cervical cancers ${ }^{65}$. Similar to cervical cancer, disruption of another viral gene, $E 2$, which acts to repress expression of $E 6$ and $E 7$ during productive infection, is frequently observed in OPSCCs that harbour integrated HPV and has been linked with an unfavourable prognosis ${ }^{66}$. The observation that the physical state of the HPV genome is of clinical significance in patients with $\mathrm{HPV}^{+} \mathrm{OPSCC}$ is consistent with these findings: an analysis of samples from 84 patients demonstrated shorter overall survival (OS) and decreased antitumour immunity in patients with gene expression from integrated HPV (those in which chimeric viralhost mRNA sequences could be detected), compared with those without evidence of integration ${ }^{67}$.

Much research has gone into understanding the molecular mechanisms by which E6 and E7 induce cell-cycle entry and DNA replication in host cells. These effects, in combination with alterations to the host genome, can result in malignant transformation of the host cell by enabling many of the hallmarks of cancer $^{68,69}$ (FIG. 2). The two best-characterized oncogenic activities of E6 and E7 involve increased degradation of p53 and Rb, respectively. The removal of these crucial tumour suppressor proteins results in loss of cell-cycle checkpoint activation in response to DNA damage and uncontrolled licensing of DNA replication, which together result in genomic instability and resistance to apoptosis ${ }^{70-75}$.

\section{Development of HPV+ OPSCC $H P V$-driven carcinogenesis}

HPVs are non-enveloped viruses with circular doublestranded DNA genomes of approximately $8 \mathrm{kbp}$. More than 200 HPV types have been identified, all of which infect and complete their productive life cycle in either the cutaneous or mucosal epithelia. Of these, the WHO currently classifies 14 mucosal HPV types (HPV-16, $18,31,33,35,39,45,51,52,56,58,59,66$ and 68 ) as 'high-risk' (hrHPV), owing to clear experimental and epidemiological evidence implicating them in cancer causation, with HPV-16 accounting for at least $85 \%$ of all $\mathrm{HPV}^{+} \mathrm{OPSCCs}^{60}$. The productive life cycle of HPV-16 is intimately linked to the terminal differentiation of keratinocytes in stratified mucosal epithelia, while carcinogenesis occurs in the context of persistent infection (postulated to be favoured in the immune-privileged microenvironment of the tonsillar crypts ${ }^{61}$ ) and is the result of an exit from productive viral replication ${ }^{62,63}$. The stepwise changes to viral and host gene expression and alterations to the host genome associated with

\section{Establishing oncogene addiction}

Inhibition of $\mathrm{Rb}$ function has long been recognized as a key oncogenic property of epigenetic reprogramming of the host cell via $\mathrm{Rb}$-independent induction of two lysine demethylases, KDM6A and KDM6B. These chromatin-modifying enzymes have broad effects on gene expression, including derepression of Homeobox (HOX) genes, which are master regulators of development and are largely silenced by polycomb group (PcG) proteins in the absence of hrHPV infection. In addition to these effects on chromatin state and derepression of PcG targets, further examples of epigenetic reprogramming by HPV include E6-dependent modulation of microRNAs and other non-coding RNAs ${ }^{76-78}$, which result in altered regulation of gene expression and modulation of DNA methylation and have been linked both to upreguation of DNA methyltransferases DNMT1 and DNMT3A in $\mathrm{HPV}^{+} \mathrm{OPSCCs}^{79}$ and to direct interaction of HPV-16 E7 with DNMT1 (REF ${ }^{80-83}$ ). Suppression of Rb function by E7 is proposed as being necessary to prevent 


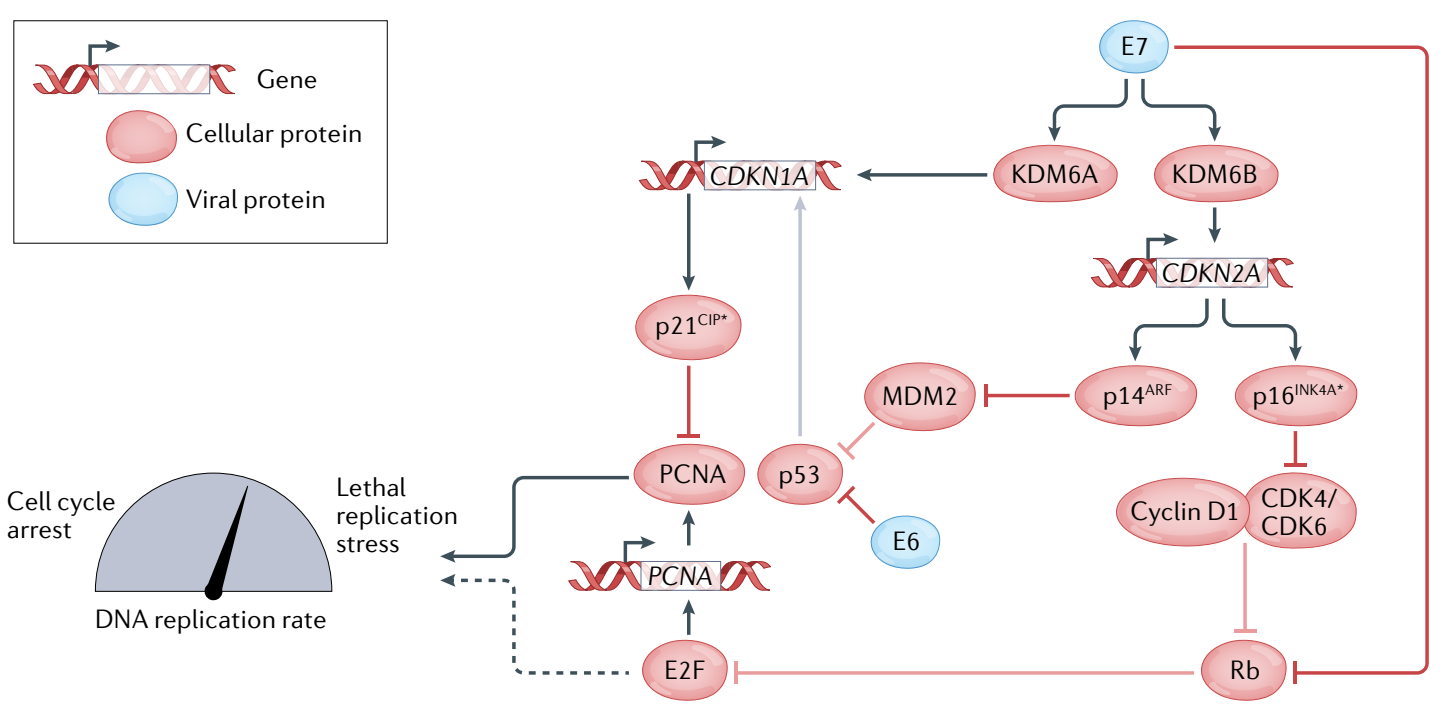

Fig. 3 | Updated model of cell-cycle perturbation by the HPV oncogenes E6 and E7. As proposed by McLaughlin-Drubin, Munger and colleagues, E7 induces the expression of lysine demethylases KDM6A and KDM6B, which in turn leads to the upregulation of CDKN1A (p21 ${ }^{\mathrm{CIP}}$ ) and CDKN2A ( $\mathrm{p} 14^{\mathrm{ARF}}$ and $\left.\mathrm{p} 16^{\mathrm{INK} 4 \mathrm{~A}}\right)$, respectively. $\mathrm{HPV}^{+}$cancer cells become dependent on the ongoing expression of $\mathrm{p} 16^{\mathrm{INK} 4 \mathrm{~A}}$ and $\mathrm{p} 21^{\mathrm{CIP}}$, with the former acting to limit CDK4/6-cyclin D activity and the latter restraining proliferating cell nuclear antigen (PCNA) activity to avoid lethal replication stress ( $\mathrm{REFS}^{83,86,87,89}$ ). Cell-cycle inhibitory proteins ( $\mathrm{p} 16^{\mathrm{INK} 4 \mathrm{~A}}$ and $\mathrm{p} 21^{\mathrm{CIP} 1}$ ), upon which human papillomavirus (HPV)-transformed cells become dependent, are starred.

induction of an oncogene-induced senescence-like response triggered by this reprogramming. The avoidance of $\mathrm{Rb}$-induced cell-cycle suppression renders HPV-transformed cells dependent on the ongoing expression of HPV oncogenes, as demonstrated by genetic loss-of-function experiments in primary cultures derived from cervical cancers ${ }^{84,85}$. This oncogene addiction has stimulated efforts to inhibit E6 and/or E7 as a therapeutic strategy, although this approach has thus far proved challenging owing to a lack of intrinsic enzymatic activity in these proteins ${ }^{86}$. However, encouraging progress has been made in exploiting the HPV oncoproteins as targets for therapeutic vaccines.

The epigenetic reprogramming of HPV-transformed cells through the E7-KDM6B axis also results in dependence on the $\mathrm{p} 16^{\mathrm{INK} 4 \mathrm{~A}}$ tumour suppressor protein (hereafter referred to as p16, one of two cell-cycle inhibitory proteins encoded by the PcG-regulated gene CDKN2A), owing to its ability to suppress CDK4 and CDK6 activity, which is required to relieve the $\mathrm{Rb}$-mediated inhibition of cell-cycle progression in uninfected cells ${ }^{87,88}$. The dependence on p16 to limit CDK4/6 activity in HPV-transformed cells is in striking contrast to many other tumour types, including oestrogen receptor-positive $\left(\mathrm{ER}^{+}\right)$breast cancer, in which CDK4/6 inhibition has proved to be an effective therapeutic strategy ${ }^{89}$. This oncogenic role of the p16 tumour suppressor highlights the cellular rewiring induced by HPV and the importance of understanding this effect for the rational design of targeted therapeutic strategies for patients with $\mathrm{HPV}^{+}$disease. The requirement of $\mathrm{p} 16$ function in HPV-transformed cells also likely explains the utility of this tumour suppressor protein as a clinical biomarker for the diagnosis of $\mathrm{HPV}^{+} \mathrm{OPSCC}$, as expression is much less likely to be lost or downregulated than that of a protein with deleterious or neutral effects on tumour cell fitness. Dependence on a second tumour suppressor protein, in this case the induction of p21CIP1 (p21 ${ }^{\mathrm{CIP} 1}$ ) expression from CDKN1A by KDM6A downstream of E7-directed epigenetic reprogramming, is needed to limit the rate of DNA replication driven by the proliferating cell nuclear antigen (PCNA) and thus avoid lethal replication stress ${ }^{90}$. The rewiring of cell-cycle control mediated by E6 and E7 (FIG. 3) also highlights that, in this updated model of HPV oncogene function, the upregulation of $\mathrm{p} 16$ seen in $\mathrm{HPV}^{+}$cancers reflects induction of KDM6B by E7 and not (as is often assumed) $\mathrm{Rb}$ inhibition ${ }^{87}$. Many other cellular proteins are targeted by the HPV oncoproteins, a comprehensive discussion of which is beyond the scope of this Review. We summarize some of these additional activities (FIG. 2) and the reader is referred to numerous detailed reviews for further information ${ }^{69,91-94}$.

\section{Somatic alterations and disease aetiology}

Despite the ability of sustained E6 and E7 expression to initiate tumorigenesis, progression to carcinoma requires the acquisition of somatic alterations in the host genome. HPV ${ }^{-}$HNSCCs typically harbour more copy-number alterations than $\mathrm{HPV}^{+} \mathrm{HNSCCs}$, suggesting a lower degree of genomic instability in the latter, while somatic variant (including single nucleotide variant (SNV) and insertion/deletion (indel)) burden seems similar between $\mathrm{HPV}^{+}$and $\mathrm{HPV}^{-} \mathrm{HNSCC}$, with a median frequency of approximately $2-3$ variants per megabase $^{95-98}$. TP53 is the most frequently mutated gene in $\mathrm{HPV}^{-}$OPSCC, occurring in at least $75 \%$ of patients; however, TP53 mutations are rarely observed in the context of $\mathrm{HPV}^{+}$disease, almost certainly owing to the inhibition of 553 function by E6 and thus an ability of the virus to phenocopy this genetic alteration ${ }^{95-99}$. However, p53 loss is not entirely equivalent to TP53 mutation, 
which can have gain-of-oncogenic function effects on the protein. Indeed, TP53 mutations are seen in a subset of heavy smokers with $\mathrm{HPV}^{+}$OPSCC and have been associated with poor prognosis in these patients ${ }^{98}$. Smoking-associated KRAS mutations typical of those seen in lung adenocarcinomas have also been reported in $\mathrm{HPV}^{+}$OPSCCs from patients with a smoking history of $>10$ pack-years ${ }^{95,98,100}$.

Somatic alterations attributable to tobacco use and ageing predominate in patients with $\mathrm{HPV}^{-}$OPSCC, whereas a high proportion of mutations in $\mathrm{HPV}^{+}$disease (at least in most patients with $\mathrm{HPV}^{+} \mathrm{OPSCC}$ who are not heavy smokers) are now thought to be caused by the off-target DNA editing activity of one or more apolipoprotein B mRNA-editing catalytic polypeptide-like (APOBEC3) enzymes, the physiological function of which is to suppress viral replication by deaminating cytosine bases in the context of single-stranded DNA or RNA ${ }^{96,97,101}$. Two of the seven human APOBEC3 enzymes (APOBEC3A and APOBEC3B) have been implicated in the cellular response to HPV infection, with evidence linking APOBEC-mediated editing of the viral genome to clearance of infection, at least in the cervix ${ }^{102}$. Sequencing of matched host exomes and viral genomes from $\mathrm{HPV}^{+}$OPSCCs suggests that off-target APOBEC activity against the host cell genome accounts for many of the somatic mutations seen in tumours in which the APOBEC response has failed to clear the virus $^{103}$ (reviewed extensively elsewhere ${ }^{104-106}$ ).

\section{Activation of PI3K signalling}

A key consequence of APOBEC activity in $\mathrm{HPV}^{+}$ OPSCCs seems to be the generation of oncogenic point mutations in PIK3CA, which encodes the p110a catalytic subunit of $\mathrm{PI} 3 \mathrm{Ka}^{96,97,101}$. Indeed, activation of the phosphoinositide 3-kinase (PI3K) signalling pathway by somatic mutation and/or copy-number alterations of PIK3CA is a key feature of $\mathrm{HPV}^{+}$OPSCC and seems to occur early in carcinogenesis ${ }^{97,107,108}$. Detection of activating mutations in PI3K components (PIK3CA, PIK3C2B and $P I K 3 R 1$ ) and their downstream mediators (such as $M T O R$ or RICTOR) or inactivating mutations in the negative regulators PTEN, TSC1 or TSC2 in metastatic tumours have all been associated with longer OS in patients with $\mathrm{HPV}^{+} \mathrm{OPSCC}^{109}$, while PIK3CA mutations have been associated with an increased risk of disease recurrence in patients with $\mathrm{HPV}^{+}$OPSCC receiving first-line chemoradiation in the setting of trials exploring de-intensification approaches ${ }^{110}$. PIK3CA mutations or amplifications have also been associated with dramatically improved outcomes in patients with HNSCC (including those with $\mathrm{HPV}^{+}$disease) requiring regular non-steroidal anti-inflammatory drugs (NSAIDs) ( $\geq 2$ days per week for at least 6 months) relative to those not taking such agents (disease-specific survival HR $0.23, P=0.0032$ and OS HR $0.31, P=0.0043$ ) in a retrospective analysis, potentially owing to increased cyclooxygenase activity in PIK3CA-altered tumours ${ }^{111}$. This intriguing observation requires confirmation in larger cohorts; nonetheless, PIK3CA mutations have also been associated with benefit from NSAIDs in patients with colorectal cancer, potentially owing to the induction of cyclooxygenase 2 activity by PI3K signalling ${ }^{112,113}$. Loss-of-function mutations in PTEN (which encodes the $\mathrm{PI}(3) \mathrm{P}_{3}$ phosphatase that reverses the reaction catalysed by class $1 \mathrm{PI} 3 \mathrm{~K}$ ) are significantly enriched in primary $\mathrm{HPV}^{+} \mathrm{OPSCCs}$, as are loss-of-function mutations in $C Y L D$, which encodes a ubiquitin ligase, and gain-of-function mutations in FGFR3, both of which can also result in activation of PI3K signalling ${ }^{97}$.

\section{Other alterations}

Genes involved in epidermal differentiation, including ZNF750, KMT2D, EP300, RIPK4 and NOTCH1, are recurrently mutated in patients with $\mathrm{HPV}^{+}$OPSCC, as are various components of the p53 (although as noted above, very rarely TP53 itself) and Rb pathways targeted by E6 and E7, including mutations in or loss of $R B 1$ (the gene encoding $\mathrm{Rb}$ ) in as many as $40 \%$ of $\mathrm{HPV}^{+}$ OPSCC $^{95,97}$. In a genomic analysis of 157 OPSCCs, 73 of which were $\mathrm{HPV}^{+}$and for which long-term clinical follow-up data were available, $\mathrm{NOTCH} 1$ mutations were associated with a significantly shorter OS duration specifically in those with $\mathrm{HPV}^{+}$disease ${ }^{98}$. This observation, together with data showing that Notch1 inactivation generates higher-grade tumours in a mouse model of HPV-16 E6/E7-driven HNSCC suggests that even though NOTCH1 expression is suppressed by E6, inactivation owing to loss-of-function mutation might have a greater effect on pathway activation and therefore lead to the development of more-aggressive tumours ${ }^{114,115}$. The importance of evading immunity to viral infection is evident also in the frequent enrichment for mutations in components of the interferon response, including DDX3X, TRAF3, IFNGR1, NFKBIA, TGFBR2, EP300 and $K M T 2 D$; these alterations are selected for despite suppression of the pathway at multiple levels by HPV oncoproteins $^{116}$.

Many of the genes that are frequently mutated in $\mathrm{HPV}^{+}$ OPSCCs encode transcriptional regulators: EP300 and $K M T 2 D$ both encode chromatin-modifying enzymes, NFKBIA encodes a negative regulator of nuclear factor $\mathrm{\kappa B}$ $(\mathrm{NF}-\mathrm{\kappa B}), D D X 3 X$ encodes a regulator of RNA metabolism and ZNF750, CASZ1 and TAF5 all encode transcription factors ${ }^{97}$. The clinical relevance of somatic alterations in these genes, together with the effects of E7 on KDM6A, KDM6B and DNMT1 discussed previously, emphasizes the importance of host cell rewiring during HPV-driven carcinogenesis. This phenomenon is evident from multiple studies that have defined gene-expression signatures for $\mathrm{HPV}^{+} \mathrm{OPSCC}$ or pan-tissue expression signatures for $\mathrm{HPV}^{+}$malignancies ${ }^{97,117,118}$.

\section{Antitumour immune responses}

MHC-loaded peptides generated as a result of nonsynonymous somatic mutations in expressed genes are the primary means by which antitumour $\mathrm{T}$ cell responses are induced in malignancies that lack a viral aetiology. Moreover, the success of immune-checkpoint inhibitors (ICIs) is associated with both the overall number of neoantigens (which is closely linked to tumour mutational burden) and their level of clonality (the fraction of tumour cells in which a given neoantigen is present) ${ }^{119,120}$. Cells that express one or more highly immunogenic 
neoantigens might be eliminated during tumour development, a process known as immunoediting ${ }^{121}$. In $\mathrm{HPV}^{+}$ cancers, all tumour cells are exquisitely dependent on the expression of the viral oncogenes $E 6$ and $E 7$, thus these proteins serve as an indispensable source of tumour-specific antigens, against which antitumour immune responses can be mounted. However, HPVs have evolved many mechanisms of immune evasion, from passive mechanisms, such as limiting the extent of infection to cells located outside of the basement membrane of the epithelium and restricting high levels of viral gene expression and viral replication to the upper layers (in which few immune cells are found), to active suppression of interferon responses and antigen presentation $^{122}$. As discussed previously, E2-mediated control of viral gene expression in the basal layer is lost during progression from persistent infection to malignancy, and invasive tumours are also able to breach the basement membrane; therefore, the active suppression of host immune responses to the virus is crucial to the ability of $\mathrm{HPV}^{+}$tumour cells to avoid immune destruction. Key mechanisms mediating this effect include selective retention of certain MHC class I components (such as HLA-A and HLA-B) in the Golgi apparatus through direct interaction with the Golgi-resident HPV-16 E5 protein, which inhibits the recognition of E5-expressing cells by $\mathrm{CD}^{+} \mathrm{T}$ cells ${ }^{123-125}$, and inhibition of MHC class I gene expression by HPV-16 E7 (REFS ${ }^{126-128}$ ).

Despite these and numerous other mechanisms through which HPV oncoproteins interfere with antigen processing and presentation (reviewed in REF.122), most $\mathrm{HPV}^{+}$OPSCCs contain evidence of ongoing intratumoural E6-specific and/or E7-specific T cell-mediated immune responses ${ }^{129,130}$. The presence of such responses seems to be strongly prognostic, with significantly improved disease-specific survival in those with HPV DNA ${ }^{+}$OPSCCs that harbour detectable HPV16 -specific T cells (HR 37.8, $P<0.001$ ). Most HPV-16specific $\mathrm{T}$ cells detected in this study were $\mathrm{CD} 4^{+}$and produced cytokines (IFN $\gamma$, TNF, IL-2 and IL-17) consistent with $\mathrm{T}$ cell polarization to an antitumour (T helper 1 $\left.\left(\mathrm{T}_{\mathrm{H}} 1\right) / \mathrm{T}_{\mathrm{H}} 17\right)$ phenotype $^{130}$. In further research, the same group implicated subsets of effector memory $\left(\mathrm{CD} 161^{+}\right)$ $\mathrm{T}$ cells with high levels of cytokine production and dendritic cells of a CD163 ${ }^{+}$subtype (DC3) as key mediators of these $\mathrm{HPV}$-specific responses in patients with $\mathrm{HPV}^{+}$ OPSCC $^{131,132}$. HPV-specific T cells have also been identified in blood from patients with $\mathrm{HPV}^{+}$OPSCCs, with circulating E7-specific $\mathrm{CD}^{+} \mathrm{T}$ cells associated with longer disease-free survival (DFS) durations ${ }^{133,134}$.

These studies of HPV-specific immune responses provide clear prognostic information, although such analyses require ex vivo culture and functional assays, which poses difficulties for translation into routine use as clinical biomarkers for predicting therapeutic responses ${ }^{135}$. Prognostic information can also be gained from less-refined analyses of the tumour immune microenvironment and circulation in patients with $\mathrm{HPV}^{+}$OPSCC. Total $\left(\mathrm{CD}^{+}\right) \mathrm{T}$ cell tumour infiltration is an independent prognostic indicator of improved OS, local progression-free survival (PFS) and distant metastasis-free survival in patients with $\mathrm{HPV}^{+}$
OPSCC $^{136,137}$. Furthermore, the extent of immune infiltration is substantially reduced in those with tumours that harbour mutational signatures attributable to tobacco smoking, offering a potential explanation for the aforementioned association between smoking and an inferior prognosis ${ }^{22,138}$. The extent of $\mathrm{T}$ cell infiltration and activation (assessed on the basis of gene-expression patterns) is also notably higher in $\mathrm{HPV}^{+}$OPSCCs than in other HPV ${ }^{+}$HNSCCs in the The Cancer Genome Atlas (TCGA) cohort, which possibly explains the greater OS benefits conferred by HPV infection in the oropharynx than in other HNSCC subsites ${ }^{139,140}$. Similarly, a comparative analysis of $\mathrm{HPV}^{+} \mathrm{OPSCC}$ and $\mathrm{HPV}^{+}$cervical cancers revealed differences in the tumour immune microenvironment related to anatomical site, with $\mathrm{HPV}^{+}$ OPSCCs harbouring a higher $\mathrm{CD}^{+}: \mathrm{CD}^{+} \mathrm{T}$ cell ratio (reflecting a higher $\mathrm{CD} 4^{+}: \mathrm{CD}^{+}$ratio in the tonsils relative to the cervical epithelium) and greater numbers of $\mathrm{CD} 4^{+} \mathrm{CD} 161^{+}$cells $^{131}$.

In addition to the DC3 cells mentioned previously, other immune cell types have also been associated with prognosis in patients with $\mathrm{HPV}^{+}$OPSCC. For example, tumour-infiltrating $\mathrm{B}$ cells are also commonly found, and $\mathrm{CD} 20^{+} \mathrm{B}$ cell infiltration is reported to be a superior prognostic marker to HPV positivity or $\mathrm{CD}^{+} \mathrm{T}$ cell infiltration ${ }^{141,142}$. Tumour-associated macrophages (TAMs) are associated with poor prognosis in many tumour types, including OPSCC; however, macrophage infiltration has been associated with improved PFS in patients with $\mathrm{HPV}^{+}$OPSCC who receive definitive chemoradiotherapy ${ }^{143}$. Skewing of macrophage polarization towards the pro-inflammatory M1 phenotype owing to high levels of IFN $\gamma$-producing T cells in $\mathrm{HPV}^{+}$OPSCC might explain this favourable association (reviewed in REF. ${ }^{144}$ ).

Upregulation of the immune-checkpoint protein $\mathrm{PD}-\mathrm{L} 1 \mathrm{~h}$ as been observed at higher frequencies in patients with $\mathrm{HPV}^{+}$than in those with $\mathrm{HPV}^{-}$OPSCC. In some patients this upregulation seems to reflect integration of the HPV genome close to the gene encoding PD-L1 (CD274) $)^{145,146}$. The increasing use of ICIs that target PD-1 or PD-L1 in patients with HNSCC will shed further light on the extent to which $\mathrm{HPV}^{+}$tumours depend on this mechanism of immune suppression. Another immune-checkpoint protein, natural killer group 2 member A (NKG2A), is expressed at higher levels in $\mathrm{HPV}^{+}$ OPSCCs in which an HPV-specific immune response can be detected and is found on tissue-resident $\left(\mathrm{CD} 103^{+}\right)$ $\mathrm{CD}^{+} \mathrm{T}$ cells, which have been linked to a favourable prognosis in patients with $\mathrm{HPV}^{+}$OPSCC and in those with other cancer types. Therapeutic anti-NKG2A antibodies are at an earlier stage of clinical development than those that target PD-1 or PD-L1, but have shown some promising results thus far (reviewed in REF. ${ }^{144}$ ).

\section{Clinical management \\ Clinical presentation and diagnosis}

OPSCC most commonly presents as a neck mass or sore throat, but might also present as dysphagia, a visualized mass, globus sensation, odynophagia or otalgia ${ }^{147}$. Most patients present with small primary tumours ( $\mathrm{T} 1$ or T2) and nodal metastases. Furthermore, the clinical 
presentation of OPSCC can be easily confused with other common benign conditions, such as laryngopharyngeal reflux or globus pharyngeus; therefore, asymptomatic neck masses should ideally be evaluated using confirmatory ultrasonography and fine-needle biopsy sampling ${ }^{148}$. OPSCCs comprise tumours located in the posterior pharyngeal wall, the soft palate, the tonsillar complex and the base of the tongue. The latter two are the most common anatomical locations and comprise $96 \%$ of oropharyngeal tumours ${ }^{20,149}$. Of note, a subset of patients with head and neck cancers will present with cervical lymphadenopathy only. These carcinomas of unknown primary are rising in incidence, most probably owing to the increasing incidence of HPV-related OPSCCs ${ }^{150}$. The presence of p16 and/or HPV DNA in metastatic lesions has been shown to indicate the oropharynx as the primary source of these tumours ${ }^{150-152}$.

In general, clinical examination per the UK National Multidisciplinary Guidelines involves direct flexible endoscopy of the upper aerodigestive tract and cross-sectional imaging ${ }^{153}$. Both PET-CT and MRI are recommended, the former for primary tumour staging and to assess the extent of soft-tissue spread, and the latter to determine the extent of nodal disease and bony invasion as well as for the detection of distant metastases to the lung and liver ${ }^{154}$. Conversely, in the USA, $\left[{ }^{18} \mathrm{~F}\right]$ fluoro-2-deoxy-D-glucose $\left({ }^{18} \mathrm{~F}\right.$-FDG $)$ PET-CT is the main modality used to assess the extent of the tumour and presence of metastases, although MRI might be used to assess the extent of local invasion.

Robust HPV testing is required to accurately discriminate between HPV positivity and negativity. A combination of immunohistochemical (IHC) staining for p16 and in situ hybridization (ISH) for high-risk HPV has demonstrated acceptable levels of both sensitivity (97\%) and specificity (94\%) and can be performed using formalin-fixed paraffin-embedded tissue $\mathrm{e}^{155}$. Current efforts to de-escalate the intensity of treatment for patients with $\mathrm{HPV}^{+} \mathrm{OPSCC}$ highlight the paramount importance of accurate determination of HPV status. The AJCC 8th edition recommends using p16 IHC only as a surrogate for HPV status because p16 positivity alone is not sufficient to detect transcriptionally active HPV in all patients. In an evaluation of the suitability of these guidelines, patients with $\mathrm{p} 16^{+} / \mathrm{HPV}^{-}$cancers had significantly lower 5-year OS than patients with $\mathrm{p} 16^{+} /$ $\mathrm{HPV}^{+}$OPSCC (33\% versus $77 \%, P<0.0001$ ). Under the new staging criteria, cancer stage was reduced in $95 \%$ of patients with $\mathrm{p} 16^{+} / \mathrm{HPV}^{-}$disease, despite these patients having a mortality rate approximately twice that of those with $\mathrm{p}^{+} 6^{+} / \mathrm{HPV}^{+}$disease on multivariate analysis (HR 2.66, 95\% CI 1.37-5.15) ${ }^{6}$. As such, a second ISH test has been recommended in the UK as standard practice by the UK Royal College of Pathologists ${ }^{6}$.

Several variant forms of SCC exist, most of which can be categorized as either keratinizing or non-keratinizing, with or without maturation (FIG. 1). Most non-keratinizing SCCs are associated with transcriptionally active high-risk HPV infection ${ }^{156}$. In this context, HPV infection increases risk, regardless of tobacco use and/or alcohol consumption. Keratinizing SCC is the most common OPSCC subtype, although only $15-25 \%$ of keratinizing
SCCs are $\mathrm{HPV}^{+}$. Histologically, these tumours resemble stratified squamous epithelium with varying degrees of architectural and cytological abnormalities, such as the formation of keratin pearls. Furthermore, the invasion pattern at the advancing front has been shown to be a clinically relevant predictor of both local recurrence and OS. Importantly, clinical presentation and histological appearance, as well as the optimal management and prognosis, vary between the different OPSCC subtypes. Other less common subtypes include basaloid SCC, papillary SCC, lymphoepithelial carcinoma, adenosquamous carcinoma, spindle-cell carcinoma and verrucous SCC. Both basaloid and papillary SCCs and lymphoepithelial carcinomas are generally associated with transcriptionally active, high-risk HPV infection in the oropharynx ${ }^{157-162}$.

In general, clinical prognostication is based upon tumour diameter and nodal status, positive surgical margins and grade of differentiation (well, moderate or poorly differentiated), including the grade of the invasive front, which involves the degree of keratinization, pleomorphism, mitotic rate, invasion pattern and patient response $^{163}$. Other independent prognostic factors for local disease recurrence and OS include invasion pattern (cohesive or non-cohesive) as well as perineural and lymphatic invasion ${ }^{164}$. Data from a retrospective study indicate no statistically significant difference in prognostic performance when measures of depth of invasion or tumour thickness are used to determine AJCC T category ${ }^{165}$. Lymph node involvement and extracapsular/ extranodal extension have been shown to have a prognostic value although some controversy remains ${ }^{166-169}$. With regard to extracapsular extension, two independent sets of investigators have reported associations between extent of extracapsular extension and OS, although a third study failed to observe a statistically significant association with OS, locoregional recurrence-free survival or distant metastasis-free survival ${ }^{170-172}$. In a cohort of patients undergoing transoral surgery and neck dissection, number of metastases was found to be an independent predictor of outcome, while extent of extracapsular spread was not ${ }^{169}$. Data from a comparison of staging systems suggest that extracapsular extension might be a surrogate of nodal volume, which itself seems to have a greater prognostic role ${ }^{167}$. The role of lymph node ratio in prognosis has also been investigated and it is associated with OS in patients with $\mathrm{HPV}^{-}$ OPSCC, although this relationship is weaker in those with $\mathrm{HPV}^{+}$disease ${ }^{173}$. The investigators suggest that the reduced prognostic relevance of lymph node ratio in $\mathrm{HPV}^{+}$disease might reflect a greater dependence on the extent of the primary tumour than on nodal spread ${ }^{173}$. Determination of the extent of extracapsular spread has generally relied on histopathological examination of surgical specimens, although CT imaging has also been recommended for use in the initial prognostic work-up. Despite this recommendation, the predictive capacity of CT remains controversial, with previous studies suggesting only moderate levels of specificity and low sensitivity, as well as poor positive and negative predictive values ${ }^{174-176}$. Nevertheless, the identification of three or more imaging criteria (irregular nodal enhancement, infiltration into adjacent tissues, indistinct nodal 
margins, matted nodes and central necrosis) has been shown to improve both specificity and positive predictive value of diagnostic CT, while the presence of necrosis has been found to be independently correlated with pathologically proven extracapsular spread ${ }^{175,176}$. More recently, contrast-enhanced CT demonstrated good sensitivity (81-85\%) with excellent interobserver agreement (weighted $\kappa=0.87$ ) in 31 patients with unknown primary HNSCCs $^{177}$. Altogether, the utility of extracapsular spread as a clinical prognostic factor, considering the challenges associated with the radiological evaluation of extranodal pathology, remains unclear. The heterogeneity of data presented thus far, including several contradictory results, warrants further large-scale multicentre studies to better guide clinical management.

The most recent (8th) edition of the AJCC staging guidelines, based on the International Collaboration on Oropharyngeal Cancer Network for Staging (ICON-S) cohort study, differentiated OPSCC on the basis of HPV status, as determined by p16 overexpression ${ }^{178}$ (TABLE 2). With changes made to the approach to $\mathrm{N}$ staging in particular, many patients with $\mathrm{HPV}^{+}$disease were assigned to a lower stage relative to previous criteria. Furthermore, this update reserves the use of the term 'stage IV' for metastatic disease only, compared with traditional staging systems, which classified locally advanced disease as stage IVa. These changes, among others, have enabled improved OS discrimination, which is especially important in the era of treatment de-intensification ${ }^{179,180}$. However, the ability to discriminate between stage groups, particularly between stages II and III and between stages III and IV, is controversial in that outcomes of certain patients with disease of a specific stage will sometimes overlap with those of patients who supposedly have disease of a more advanced stage. Thus, implementation of the staging system in clinical practice requires further adaptation to include consideration of other relevant prognostic factors ${ }^{181,182}$.

\section{Table 2 | Important differences between AJCC TNM 7th and 8th editions}

\begin{tabular}{|c|c|c|}
\hline TNM stage & $\mathrm{p} 16^{-}$ & $\mathrm{p} 16^{+}$ \\
\hline \multirow[t]{3}{*}{ Tstaging } & \multirow[t]{3}{*}{ Same as AJCC 7th edn } & Tis: not included \\
\hline & & $\begin{array}{l}\text { T0: only for } \mathrm{p} 16^{+} \text {metastatic lymph } \\
\text { nodes }\end{array}$ \\
\hline & & $\begin{array}{l}\text { T4: formerly divided into T4a and } \\
\text { T4b, now unified into a single } \\
\text { category }\end{array}$ \\
\hline \multirow{4}{*}{$\begin{array}{l}\text { Clinical N } \\
\text { staging }\end{array}$} & \multirow{4}{*}{$\begin{array}{l}\text { N3: nodes }>6 \mathrm{~cm} \text { in diameter } \\
\text { further subdivided into N3a and } \\
\mathrm{N} 3 \mathrm{~b} \text { on the basis of the absence } \\
\text { (former) or presence (latter) of } \\
\text { extranodal extension }\end{array}$} & $\mathrm{N} 1$ : ipsilateral lymph nodes $\leq 6 \mathrm{~cm}$ \\
\hline & & $\begin{array}{l}\text { N2: bilateral or contralateral } \\
\text { nodes } \leq 6 \mathrm{~cm}\end{array}$ \\
\hline & & no N2 subcategories \\
\hline & & N3: nodes $>6 \mathrm{~cm}$ \\
\hline \multirow[t]{3}{*}{$\begin{array}{l}\text { Pathological } \\
\text { N staging }\end{array}$} & \multirow[t]{3}{*}{ Same as AJCC 7th edn } & $\begin{array}{l}\text { N1: involvement of } \leq 4 \text { metastatic } \\
\text { lymph nodes }\end{array}$ \\
\hline & & N2: $>4$ metastatic nodes \\
\hline & & N3: removed \\
\hline HPV status & \multicolumn{2}{|c|}{$\begin{array}{l}\text { p16 testing; tumours with at least moderate staining intensity } \\
\text { and diffuse staining ( } \geq 75 \% \text { of tumour cells) classified as probable } \\
\text { HPV-associated aetiology on the basis of p16 positivity }\end{array}$} \\
\hline
\end{tabular}

See REF. ${ }^{244}$. AJCC, American Joint Committee on Cancer; HPV, human papillomavirus.
Importantly, a subgroup of patients with $\mathrm{p} 16^{+}$but HPV DNA- OPSCCs exists, with a significantly worse prognosis than patients with HPV DNA ${ }^{+}$disease ${ }^{6,183}$. Therefore, and as mentioned previously, determination of HPV status should involve assessments of both p16 (via IHC) and high-risk HPV DNA (using ISH). Other tumour-specific and patient-specific factors might also be necessary considerations for attempts to improve prognostication. In a recursive partitioning study (0129) conducted by the Radiotherapy Oncology Group (RTOG), low, intermediate and high risk groups were established based on HPV status, tobacco exposure and extent of lymph node invasion ${ }^{22}$. Patients deemed to be low risk in this study had $\mathrm{HPV}^{+}$disease with low tobacco exposure or a history of smoking $\leq 10$ pack-years in addition to only one ipsilateral lymph node with a diameter $<6 \mathrm{~cm}$; intermediate-risk patients have $\mathrm{HPV}^{+}$ disease, a history of smoking of $>10$ pack-years and advanced lymph node disease (multiple ipsilateral lymph nodes, one or more contralateral lymph nodes or any lymph node measuring $>6 \mathrm{~cm}$ ) or $\mathrm{HPV}^{-}$disease with low tobacco exposure and $<\mathrm{T} 4$ stage tumour; high-risk patients had $\mathrm{HPV}^{-}$disease with a history of smoking of $>10$ pack-years or stage T4 disease. A retrospective analysis assessing the 5-year survival outcomes of this cohort demonstrated the robustness of this stratification, with persistent differences observed in both PFS and $\mathrm{OS}^{184}$. Taking into account a second, independent cohort (RTOG-0522), combined 5-year PFS for patients with low-risk, intermediate-risk or high-risk disease were $72.9 \%, 56.1 \%$ and $42.2 \%$, respectively, with 5 -year OS of $88.1 \%, 69.9 \%$ and $45.1 \%$. On the basis of these data, the authors recommended therapeutic de-intensification for patients in the low-risk group.

Crucially, an analysis of the US National Cancer Database found anatomical subsite to be an independent prognostic factor ${ }^{149}$. However, the current AJCC guidelines, although stratifying for HPV status, do not consider subsite. Despite this lack of guideline recognition, anatomical location remains important because SCCs of the tonsils or base of the tongue are more frequently $\mathrm{HPV}^{+}$than those at other sites. Indeed, the prevalence of HPV infection in these sites seems to be lower, with approximately $19-22 \%$ of tumours testing positive for HPV, compared with 56-70\% for OPSCCs located in the tonsils or base of the tongue ${ }^{185,186}$. Furthermore, the prognostic value of HPV infections outside these two anatomical locations seems to be less robust, calling into question the appropriateness of the current AJCC staging system for SCCs at these sites ${ }^{186}$. These considerations warrant continued investigation of more comprehensive, and potentially more accurate, prognosticators that incorporate the effects of subsite as well as patient history, with particular regard to smoking history as discussed above, on top of current AJCC staging.

\section{Treatment and follow-up}

The treatment of patients with OPSCC typically involves surgical excision, primary radiotherapy or chemoradiotherapy ${ }^{153}$ (see TABLE 3 for UK recommendations). Historically, surgical excision has involved open surgery; however, owing to concerns of cosmetic and/or 
Table 3 | UK/US treatment recommendations for HPV+ OPSCC (not yet updated for AJCC 8th edition staging guidelines)

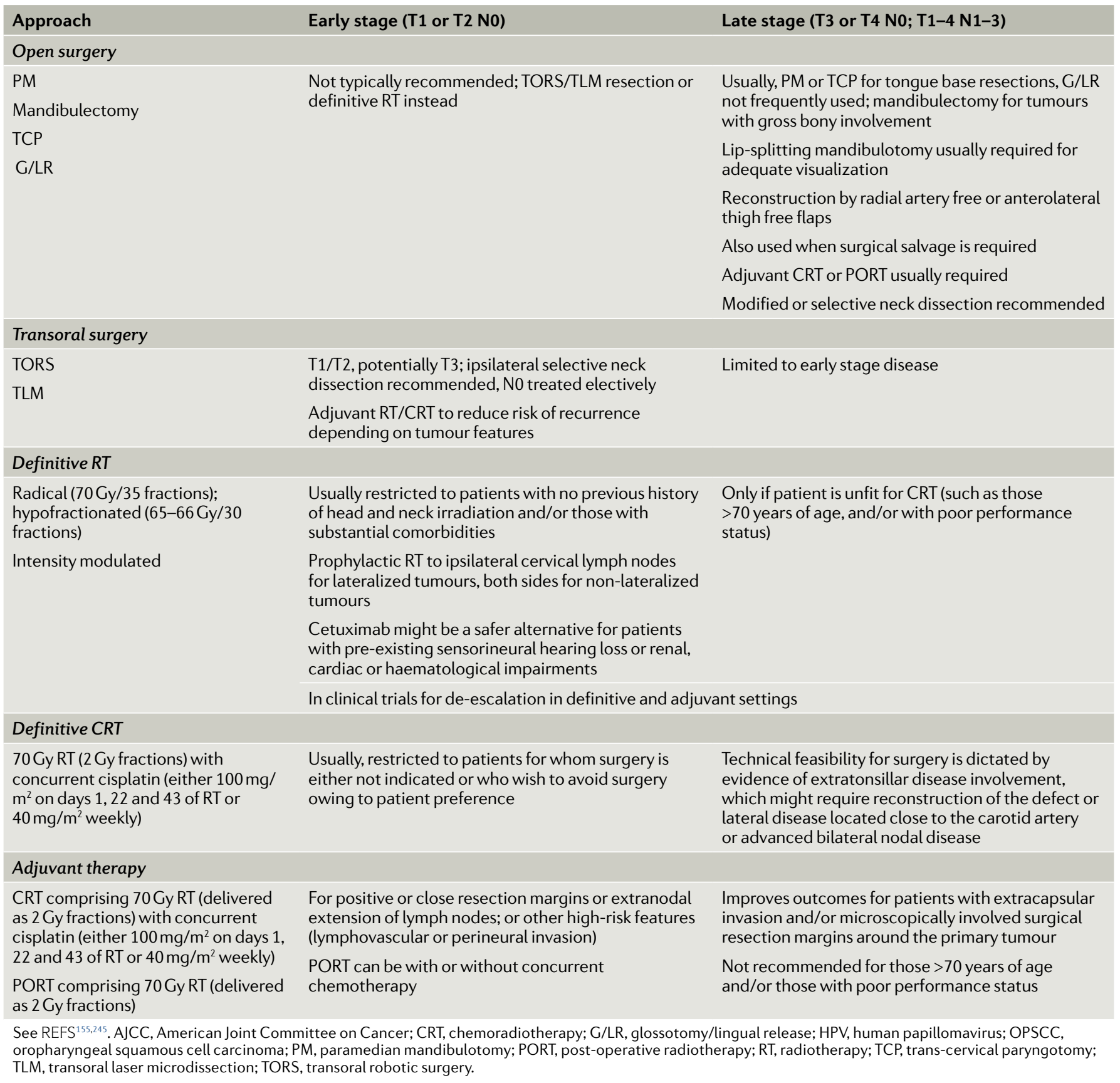

functional morbidities, for patients with early stage disease this has largely been replaced by less-invasive techniques, such as transoral laser microsurgery (TLMS) and transoral robotic surgery (TORS). Primary radiotherapy and chemoradiotherapy are also widely used according to patient preference, or if TLMS or TORS is not technically feasible, such as owing to a large (T3 or above) primary tumour diameter, poor transoral access or advanced bilateral nodal disease. The current standard of care consists of 66-70 Gy radiotherapy with concurrent platinum-based chemotherapy, typically cisplatin based (TABLE 4; Supplementary Information).

Despite the favourable prognosis associated with $\mathrm{HPV}^{+}$OPSCC, $10-25 \%$ of patients will develop disease recurrence, the majority within 2 years but some up to 5 years after initial diagnosis. Thus, a robust and effective monitoring protocol for patients with $\mathrm{HPV}^{+} \mathrm{OPSCC}$ who are in remission is crucial. Typical follow-up monitoring involves regular clinical examination. The National Comprehensive Cancer Network recommends examinations every 1-3 months in the first year, then every 2-6 months in the second year, every 4-8 months up to year 5 and then subsequently once per year ${ }^{187}$. However, even with regular clinical examinations, the ability to detect disease recurrence is limited.

HPV DNA has been shown to be a useful biomarker for the monitoring of post-treatment disease status. In a prospective study with results published in 2019, 
continued detection of HPV DNA (of the same type as detected originally in tumour specimens) in oral rinses following completion of treatment was predictive of locoregional recurrence and unfavourable 2-year OS. Although the predictive value of HPV DNA for distant metastasis was weaker, the authors suggested that oral and plasma HPV DNA detection could potentially be combined to provide an effective biomarker of treatment response and risk of disease progression ${ }^{188}$. Furthermore, measuring circulating HPV DNA in plasma samples has proved an extremely sensitive method of detecting disease recurrence ${ }^{189}$. In a study involving 115 patients, two consecutive positive tests had a positive predictive value of $94 \%$ and a negative predictive value of $100 \%$. Therefore, this approach might enable earlier detection of recurrence and, as a result, improve the efficacy of salvage treatment thereafter ${ }^{189}$.

Outcomes with primary TORS/TLMS with or without adjuvant chemoradiotherapy. Until recently ( 2010), OPSCC was generally treated with primary radiotherapy (plus or minus chemotherapy) owing to the substantial risk of morbidities associated with open surgery. However, owing to advances in surgical technology, minimally invasive approaches (such as TORS or TLMS) have become the mainstay of treatment for patients with early stage OPSCC. Patients with advanced-stage OPSCC, or with disease features not amenable to TORS or TLMS, typically receive chemoradiotherapy, with surgical salvage available for those with disease progression $^{190}$. A prospective study that assessed the efficacy of TLMS in predominantly early-stage (I or II) disease demonstrated impressive 5-year survival outcomes, with DFS, disease-specific survival and OS of $85 \%, 93 \%$ and $90 \%$, respectively. The recurrence rate was $12 \%$, mainly due to the emergence of distant metastases, and $90 \%$ of recurrences occurred within the first 2 years. Minimal post-treatment morbidities were observed, and in the absence of indications for gastrostomy, only $4 \%$ of patients had a gastrostomy tube ${ }^{191}$.

Importantly, most patients with OPSCC treated with TORS or TLMS receive adjuvant radiotherapy, and a minority will also receive chemotherapy ${ }^{192}$. As such, appropriate risk stratification is needed to safely de-escalate adjuvant therapy and thus capitalize on the reduced incidence of post-treatment morbidities offered by minimally invasive surgical techniques. Data from two independent studies demonstrate that adjuvant therapy lowers the risk of local and regional recurrence; however, no statistically significant differences in OS have been observed owing to high salvage rates ${ }^{193,194}$. Indeed, even patients who receive upfront adjuvant therapy might have disease relapse, although salvage treatments are generally successful, resulting in excellent survival outcomes. This consideration is especially important owing to the various toxicities associated with adjuvant radiotherapy or chemoradiotherapy. For example, in one study, a greater risk of gastrostomy was observed in patients who received adjuvant therapy ${ }^{193}$. Indeed, patients who received TORS alone are reported to have superior quality of life (QOL) and functional outcomes at 6 months, most probably reflecting avoidance of the adverse effects of adjuvant therapy, including xerostomia, odynophagia and oral thrush, that are detrimental to patient-reported outcomes ${ }^{195}$.

When adjuvant radiotherapy is indicated, reducing the radiation dose in patients with favourable risk factors (such as negative surgical margins and/or early stage disease) can ameliorate the risk of treatment-associated morbidities while maintaining efficacy. For example, reducing adjuvant radiation dose from $60-66$ Gy to $30-36$ Gy in patients with negative margins and a minimal smoking history has been shown to lead to improved swallowing and overall QOL outcomes while maintaining excellent 2-year locoregional control, PFS and OS $(96.2 \%, 91.1 \% \text { and } 98.7 \% \text {, respectively })^{196}$. Alternatively,

Table 4 | Clinical trials investigating the efficacy of induction therapy in HPV+ OPSCC

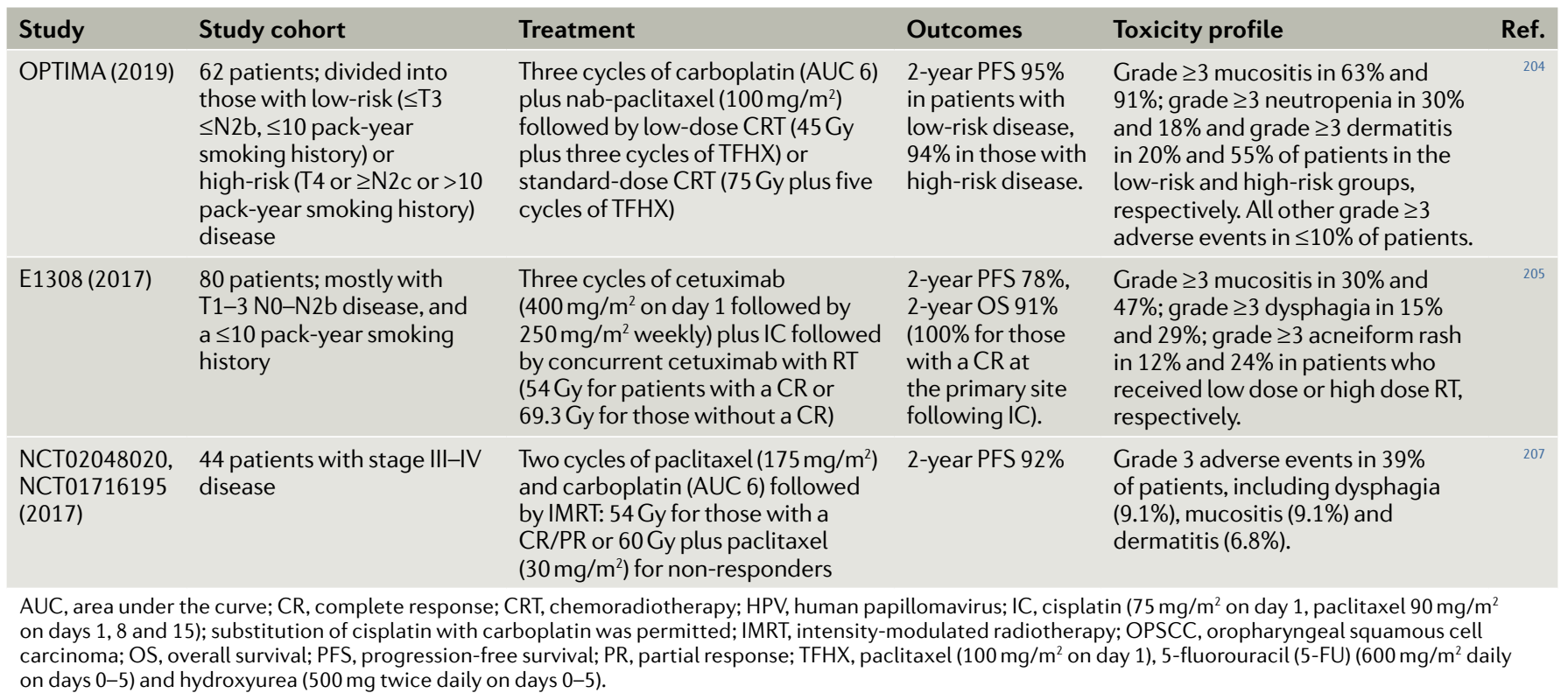


Table 5 | Trials investigating de-escalation or replacement of chemotherapy and/or radiotherapy in $\mathrm{HPV}^{+}$OPSCC

\begin{tabular}{|c|c|c|c|c|c|}
\hline Study & Study cohort & Treatment & Outcomes & Toxicity profile & Ref. \\
\hline MC1273 (2019) & $\begin{array}{l}80 \text { patients with } \leq 10 \\
\text { pack-year smoking } \\
\text { history, negative margins; } \\
\text { cohort B included } \\
\text { patients with extranodal } \\
\text { extension }\end{array}$ & $\begin{array}{l}\text { Cohort A: } 30 \text { Gy RT plus docetaxel } \\
\left(15 \mathrm{mg} / \mathrm{m}^{2}\right) \\
\text { Cohort B: extranodal extension } \\
\text { to } 36 \mathrm{~Gy}\end{array}$ & $\begin{array}{l}\text { 2-year locoregional } \\
\text { tumour control } \\
96.2 \%, \text { PFS } 91.1 \% \text {, OS } \\
98.7 \%\end{array}$ & $\begin{array}{l}\text { Grade } \geq 3 \text { toxicities before RT } \\
\text { in } 2.5 \% \text { of patients, no grade } \geq 3 \\
\text { toxicities at } 1 \text { or } 2 \text { years after RT }\end{array}$ & 196 \\
\hline $\begin{array}{l}\text { NCT01530997 } \\
(2015)\end{array}$ & $\begin{array}{l}43 \text { patients with T0-3 } \\
\text { N0-2c M0 disease and a } \\
\text { minimal smoking history }\end{array}$ & $\begin{array}{l}60 \text { Gy IMRT with concurrent } \\
\text { cisplatin }\left(30 \mathrm{mg} / \mathrm{m}^{2}\right)\end{array}$ & $\begin{array}{l}\text { 3-year locoregional } \\
\text { control } 100 \% \text {, distant } \\
\text { MFS } 100 \% \text {, DSS } 100 \%\end{array}$ & $\begin{array}{l}\text { Grade } \geq 3 \text { dysphagia in } 39 \%, \\
\text { grade } \geq 3 \text { mucositis in } 35 \% ; \\
\text { chemotherapy-related } \\
\text { grade } \geq 3 \text { toxicities included } \\
\text { haematological events ( } 11 \%) \\
\text { nausea }(18 \%) \text { and vomiting ( } 5 \%)\end{array}$ & 201 \\
\hline $\begin{array}{l}\text { Quarterback and } \\
\text { Quarterback 2b } \\
\text { (2021) }\end{array}$ & $\begin{array}{l}24 \text { and } 65 \text { patients; stage } \\
\text { III/IV disease without } \\
\text { distant metastases (per } \\
\text { AJCC 7th edn staging) }\end{array}$ & $\begin{array}{l}\text { Quarterback: three cycles } \\
\text { of induction chemotherapy; } \\
\text { responders randomized 2:1 to } \\
\text { receive 56 Gy (rdCRT) or 70 Gy } \\
\text { (sdCRT) RT with concurrent } \\
\text { carboplastin (AUC 1.5) } \\
\text { Quarterback 2b: 56/50.4 Gy IMRT }\end{array}$ & $\begin{array}{l}\text { Combined rdCRT } \\
\text { arms: 2-year LRC, PFS } \\
\text { and OS } 87.4 \%, 84.4 \% \\
\text { and } 90.6 \%\end{array}$ & $\begin{array}{l}\text { No therapy-related } \\
\text { mortality, minimal long-term } \\
\text { consequences (to be reported) }\end{array}$ & 246 \\
\hline ORATOR2 (2021) & $\begin{array}{l}61 \text { patients with stage } \\
\text { T1-2 N0-2 (AJCC 8th edn) } \\
\text { tumours }\end{array}$ & $\begin{array}{l}\text { De-intensified IMRT } \\
\text { ( } 60 \mathrm{~Gy} \pm \text { chemotherapy) vs TORS } \\
\text { plus ND ( } \pm \text { adjuvant } 50 \text { Gy IMRT) }\end{array}$ & $\begin{array}{l}\text { Estimated } 2 \text {-year OS } \\
100 \% \text { vs } 89.2 \% \text { in the } \\
\text { IMRT vs TORS plus } \\
\text { ND arms, respectively }\end{array}$ & $\begin{array}{l}\text { Grade } 2-5 \text { toxicities in } 67 \% \\
\text { of patients in the RT arm and } \\
71 \% \text { in the TORS plus ND arm. } \\
\text { Study terminated early owing } \\
\text { to treatment-related mortality } \\
\text { and unacceptable PFS in the } \\
\text { TORS plus ND arm }\end{array}$ & 247 \\
\hline
\end{tabular}

An overview of ongoing trials is provided in Supplementary Information. AUC, area under the curve; AJCC, American Joint Committee on Cancer; CRT, chemoradiotherapy; DSS, disease-specific survival; ECOG, Eastern Co-operative Oncology Group; HPV, human papillomavirus; IMRT, intensity-modulated radiotherapy; LRC, locoregional control; MDADI, MD Anderson Dysphagia Inventory; MFS, metastasis-free survival; ND, neck dissection; OPSCC, oropharyngeal squamous cell carcinoma; QOL, quality of life; PFS, progression-free survival; PS, performance status; rdCRT, reduced-dose chemoradiotherapy; RT, radiotherapy; sdCRT, standard-dose chemoradiotherapy; TORS, transoral robotic surgery.

data from the AVOID study demonstrate that avoiding irradiation of the resected primary tumour site and only targeting at-risk neck areas with reduced radiation doses in patients with early stage disease might be safe and can also result in high 2-year local control rates and OS (98.3\% and $100 \%$, respectively).

The safety and efficacy of de-intensified adjuvant therapy following TORS is being evaluated further in several ongoing trials, such as PATHOS and ECOG3311 $\left(\right.$ REF. $\left.{ }^{197}\right)$. An update from ECOG3311 demonstrates that primary TORS and reduced post-operative radiotherapy without chemotherapy provide excellent oncological outcomes at a follow-up duration of 35 months, with favourable QOL and functional outcomes, in patients with intermediate-risk $\mathrm{HPV}^{+} \mathrm{OPSCC}^{198-200}$. Data from the SIRS and MINT trials (NCT02072148 and NCT03621696, respectively), are expected to further confirm the accuracy of pathological characteristics (such as extracapsular spread, lymphovascular invasion, perineural invasion, surgical margins and tumour stage) for the allocation of treatment, with particular regard for the omission of adjuvant therapy in patients with low-risk disease. The feasibility of reduced-dose adjuvant radiotherapy in patients with high-risk disease will also be further investigated in both DART-HPV
(NCT02908477) and DELPHI (NCT03396718) (TABLE 5, Supplementary Information).

Outcomes with primary chemoradiotherapy. Despite the positive results obtained with minimally invasive surgical approaches, primary radiotherapy and/or chemoradiotherapy are still widely used. Over the past 10 years, efforts to de-escalate radiation dose have demonstrated both excellent oncological outcomes and improved morbidity rates. Data from two studies demonstrate high pathological response rates to reduced-dose intensity-modulated radiotherapy (IMRT) with concurrent low-dose cisplatin for patients with early stage disease ${ }^{201,202}$. Excellent 3-year local and regional control rates were observed with a 3 -year OS of $95 \%{ }^{203}$. For patients with advanced-stage disease (stages III-IV), induction chemotherapy followed by reduced-dose chemoradiotherapy has proved to be a promising approach in terms of reducing the risks of treatment-associated morbidities while maintaining acceptable survival rates ${ }^{204-207}$. Indeed, those who respond well to induction chemotherapy are more likely to have radiosensitve tumours, which could enable improvements in both oncological and long-term functional outcomes such as swallowing, nutritional status and overall QOL. 
Table 6 | Trials investigating de-escalation of adjuvant therapy in $\mathrm{HPV}^{+}$OPSCC

\begin{tabular}{|c|c|c|c|c|c|}
\hline Study & Cohort & Treatment & Outcomes & Toxicity profile & Ref. \\
\hline $\begin{array}{l}\text { SIRS } \\
(2021)\end{array}$ & $\begin{array}{l}54 \text { patients with stage I, II, III } \\
\text { and intermediate stage IVa } \\
\text { (T1 N0-2b, T2 N0-2b, AJCC 8th } \\
\text { edn) disease, with stratification } \\
\text { based on pathological prognosis } \\
\text { (based on ECS, LVI, PNI) }\end{array}$ & $\begin{array}{l}\text { TORS with follow-up monitoring } \\
\text { for patients with a good prognosis } \\
\text { (group 1); reduced-dose adjuvant } \\
\text { RT or CRT based on risk status for } \\
\text { patients with a poor prognosis } \\
\text { (group } 2 \text { or } 3 \text { ) }\end{array}$ & $\begin{array}{l}\mathrm{mPFS} 91.3 \%, 86.7 \% \text { and } \\
93.3 \% \text { for groups } 1-3 \text {, } \\
\text { respectively, at a median } \\
\text { follow-up duration of } \\
43.9 \text { months }\end{array}$ & $\begin{array}{l}\text { Group 1: dysphagia in } 37 \% \text {, severe } \\
\text { pain in } 29.6 \% \text {, anxiety in } 11.1 \% \text {; } \\
\text { group 2: altered taste/dysgeusia } \\
\text { in } 100 \% \text {, xerostomia in } 66.6 \% \text { and } \\
\text { severe pain in } 66.6 \% \text {; group 3: } \\
\text { dysphagia in } 100 \% \text {; pain in } 100 \% \text {; } \\
\text { dysarthria in } 50.0 \%\end{array}$ & 248 \\
\hline $\begin{array}{l}\text { AVOID } \\
(2020)\end{array}$ & $\begin{array}{l}60 \text { patients with pT1-pT2 } \\
\text { N1-3 disease with favourable } \\
\text { prognostic features underwent } \\
\text { TORS at the primary site }\end{array}$ & $\begin{array}{l}\text { Adjuvant RT omitting the } \\
\text { tumour bed }\end{array}$ & $\begin{array}{l}\text { 2-year local control } \\
98.3 \% ; 2 \text {-year OS } 100 \%\end{array}$ & $\begin{array}{l}\text { AEs in } 30 \% \text { : including radiation } \\
\text { dermatitis ( } 13.33 \%) \text {, oral mucositis } \\
(5.00 \%) \text { and dysphagia ( } 3.33 \%)\end{array}$ & 249 \\
\hline
\end{tabular}

An overview of ongoing trials is provided in Supplementary Information. AEs, adverse events; AJCC, American Joint Committee on Cancer; CRT, chemoradiotherapy; ECS, extracapsular spread; HPV, human papillomavirus; IMRT, intensity-modulated radiotherapy; LVI, lymphovascular invasion; mPFS, median progression-free survival; OPSCC, oropharyngeal squamous cell carcinoma; OS, overall survival; PNI, perineural invasion; PFS, progression-free survival; RT, radiotherapy; TORS, transoral robotic surgery.

With regard to the necessity of administering chemotherapy alongside radiotherapy, results from one study show that radiotherapy alone might be sufficient for patients with locally advanced $\mathrm{HPV}^{+}$disease. Indeed, relative to chemoradiotherapy, radiotherapy alone is less effective in patients with $\mathrm{p} 16^{-} / \mathrm{HPV}^{\mathrm{DNA}} \mathrm{N}^{-}$OPSCC, although no statistically significant difference in survival outcomes exists for those with $\mathrm{p} 16^{+} / \mathrm{HPV} \mathrm{DNA}{ }^{+}$ disease $^{208}$. However, in addition to HPV status, the extent of disease might be an additional important factor when considering the exclusion or de-escalation of chemotherapy. In a retrospective analysis of data from more than 600 patients, concurrent chemoradiotherapy reduced the risk of metastatic disease in patients with high-risk (AJCC 7th edition T4 and/or N3) $\mathrm{HPV}^{+}$ OPSCC but not in those with low-risk disease ${ }^{209}$. Conversely, the addition of concurrent cisplatin led to improved DFS in a phase II trial involving patients with low-risk $\mathrm{HPV}^{+}$OPSCC, in comparison with those who received radiotherapy alone ${ }^{210}$. Owing to these differing results, reliable conclusions regarding the safety and efficacy of excluding chemotherapy from the primary treatment of patients with advanced-stage $\mathrm{HPV}^{+}$ OPSCC cannot be drawn.

At present, the pursuit of treatment de-escalation should remain within the confines of a well-designed clinical trial, as recommended in an American Society for Radiation Oncology (ASTRO) consensus paper from 2017 $\left(\mathrm{REF}^{211}\right)$. Nonetheless, ongoing and future studies might provide the necessary evidence to update the current standard of care (TABLE 6, Supplementary Information). These include the EVADER trial (NCT03822897) for patients with early stage disease, which aims to determine survival outcomes with reduced-dose radiotherapy with or without concurrent chemotherapy. The safety and efficacy of hypofractionated radiotherapy with concurrent chemotherapy as well as that of stereotactic ablative radiotherapy boost and de-escalated chemoradiotherapy will be further investigated in HYHOPE (NCT04580446) and SHORT-OPC (NCT04178174), respectively.
Furthermore, the Quarterback trials (NCT01706939 and NCT02945631) aim to determine the safety and efficacy of reduced-dose radiotherapy in patients with advanced-stage disease (stages III-IV). Results from these trials and others will enable a better and more comprehensive understanding of the feasibility of de-escalated primary radiotherapy or chemoradiotherapy and will hopefully provide the necessary evidence to influence the current standard of care.

TORS or primary radio/chemoradiotherapy. A retrospective query of the National Cancer Database failed to reveal any statistically significant difference in OS in patients with $\mathrm{HPV}^{+}$OPSCC who received either primary TORS or primary radiotherapy ${ }^{192}$. Survival outcomes might be similar between the two methods, although differences in their respective toxicity profiles and the risks of consequent morbidities are important considerations in the clinical decision-making process.

Importantly, before the ORATOR trial, no data from prospective studies investigating differences in outcome between patients who received TORS/TLMS alone and those who received primary chemoradiotherapy were available $^{212}$. The ORATOR trial was unable to determine definitive differences in survival outcomes between patients receiving these two treatment modalities owing to its modest sample size, with similar QOL outcomes observed in addition to a spectrum of treatment-specific toxicities ${ }^{213}$. However, the trial investigators only reported 1-year swallowing and oncological outcomes data. Importantly, the authors observed a risk of bleeding associated with TORS, although multi-institutional approaches to TORS with large patient numbers showed only low rates of severe bleeding ${ }^{214,215}$. As such, patients should currently be presented with both treatment options, including discussions of the relative risks and benefits associated with each approach. A second study, ORATOR2 (NCT03210103), is currently underway and is expected to further confirm these findings and determine OS outcomes in a larger cohort of patients. 
Targeted therapies. Several ongoing clinical trials are investigating the efficacy of targeted therapy in combination with the various other available treatment modalities in a range of treatment settings. The anti-EGFR monoclonal antibody cetuximab has been investigated as an alternative to cisplatin in an effort to reduce the risks of treatment-related toxicities and morbidities in two prospective studies. The adverse event profile was similar in both groups of patients, although those receiving cetuximab had inferior locoregional disease control with an increased incidence of distant metastases; furthermore, reductions in both PFS and OS were observed $^{216,217}$. EGFR is amplified in the majority of head and neck cancers, although an important difference in expression pattern is likely to exist in oropharyngeal cancers, specifically ${ }^{218}$. Genomic studies have failed to demonstrate the selection of clones harbouring mutated or amplified EGFR in patients with $\mathrm{HPV}^{+}$tumours, in contrast to those with $\mathrm{HPV}^{-}$tumours. However, EGFR has been shown to be upregulated through gene fusion $^{95,219}$.

Data from one study demonstrate the safety of an induction chemotherapy regimen consisting of deintensified chemotherapy in combination with the antiviral agent ribavirin and the EGFR/HER2 inhibitor afatinib in patients with locally advanced $\mathrm{HPV}^{+}$ OPSCC $^{220}$. Biologically, the investigators postulate that the antitumour effects of afatinib are mediated by inhibition of HER2 signalling, which is oncogenically dysregulated through the action of the E6 protein. This approach seems promising, although further investigation is needed to better understand the biological mechanisms of this combination as well as its efficacy as an alternative, de-intensified induction therapy approach.

Emergence of immunotherapies. Promoting de novo or potentiating pre-existing antitumour immune responses to viral antigens (particularly those derived from E6 and E7) in patients with $\mathrm{HPV}^{+}$malignancies is a tantalizing and long-sought prospect for immunotherapy. The many and varied approaches to immunotherapy for $\mathrm{HPV}^{+}$cancer that have been developed over the past 20 years are covered in detail elsewhere ${ }^{221}$; here, we highlight data from selected clinical trials involving patients with $\mathrm{HPV}^{+} \mathrm{OPSCC}$, although we emphasize that, thus far, only anti-PD-1/PD-L1 antibodies have been approved for clinical use $\mathrm{e}^{221-223}$.

The anti-PD-1 antibodies nivolumab and pembrolizumab were first approved by the FDA for patients with metastatic, platinum-refractory HNSCC (regardless of HPV status) in 2016, on the basis of data from the phase III trials CheckMate-141 and KEYNOTE-040, respectively. Pembrolizumab was also approved as a first-line monotherapy for patients with PD-L1 ${ }^{+}$ (combined-positive score $\geq 1 \%$ ) metastatic or unresectable HNSCC in 2019, based on data from the phase III KEYNOTE-048 trial ${ }^{224-226}$. These trials all included patients with both $\mathrm{HPV}^{+}$and $\mathrm{HPV}^{-}$disease; therefore, several systematic reviews have since investigated possible associations between HPV status and outcomes, with three studies suggesting increased objective response rates (ORRs) and improved OS in patients with $\mathrm{HPV}^{+}$ disease $^{227-229}$, one of which ${ }^{227}$ suggests a stronger relationship in the context of PD-L1 blockade, whereas another ${ }^{230}$ found no association between HPV status and outcome. All four studies highlight the need for further research into this important question and point to a lack of data on the relationship between HPV status and PFS in patients receiving adjuvant anti-PD-1/PD-L1 antibodies. In this regard, the anti-PD-L1 antibody atezolizumab is currently being tested in a phase III trial as monotherapy in the adjuvant setting, following definitive treatment for locally advanced $\mathrm{HPV}^{+}$(stage III) or $\mathrm{HPV}^{-}$(stage IVA and IVB) HNSCC ${ }^{231}$.

A phase Ib trial combining nivolumab and stereotactic body radiation therapy (SBRT) in the neoadjuvant setting resulted in a high pathological complete response (pCR) rate $(67 \%)$ among a cohort of 21 patients with locally advanced HNSCC (NCT03247712), 16 of whom had $\mathrm{HPV}^{+}$disease ${ }^{232}$. The investigators noted the high rate of major pathological responses (MPRs) to radiotherapy alone among patients with $\mathrm{HPV}^{+}$disease in this trial, indicating a need to determine the separate contribution of each modality to these reponses. These investigators also noted the unsuitability of radiographic response as an indicator of pathological response in this context, given the short treatment window of 6 weeks. The combination of the anti-PD-L1 antibody durvalumab with SBRT is currently being tested in NCT03618134, a phase Ib/II trial specifically involving patients with $\mathrm{HPV}^{+}$OPSCC. The role of nivolumab monotherapy has also been investigated in the neoadjuvant setting in CheckMate-358 (REF. ${ }^{232}$ ), with low radiographic response rates observed both in patients with $\mathrm{HPV}^{+}(12 \%)$ and in those with $\mathrm{HPV}^{-}(8.3 \%)$ disease ${ }^{233}$. No pCRs were seen in this study and among patients with $\mathrm{HPV}^{+}$disease, only one of 17 patients evaluated had an MPR, with three more having partial pathological responses. In the phase Ib CIAO trial involving 28 patients with OPSCC, of whom 24 had p16 tumours, 29\% had an MPR and no increased benefit was observed from addition of the anti-CTLA4 antibody tremelimumab ${ }^{234}$. As noted elsewhere ${ }^{232}$, however, differences in the criteria used to assess pathological response make comparisons of data from these different neoadjuvant trials difficult, and overall, the pathological response rates of patients with HNSCC who received neoadjuvant anti-PD-1/PD-L1 antibodies as monotherapies appear to be lower than those seen in several other cancer types ${ }^{233}$.

Therapeutic vaccines based on E6 and/or E7 have long been investigated as treatments for cervical cancer; unfortunately thus far without any notable clinical success. Several therapeutic vaccines targeting E6 and/or E7 have entered trials for patients with $\mathrm{HPV}^{+} \mathrm{OPSCC}$, with numerous studies now investigating combinations with an ICI or another immunomodulatory agent ${ }^{223,235}$. Of the few trials with outcomes data available, an ORR of $36 \%$ and median OS duration of 17.5 months were observed in 22 patients with $\mathrm{HPV}^{+}$OPSCC in a phase II trial combining nivolumab with an HPV-16 E6/E7 peptide vaccine (ISA 101), which compares favourably with data from trials testing nivolumab monotherapy ${ }^{236}$. MEDI0457 (a DNA vaccine encoding E6 and E7 antigens 
from HPV-16 and HPV-18, administered together with DNA encoding IL-12 as an adjuvant) induced durable $\mathrm{HPV}$-specific immune responses in 18 of 21 patients with locally advanced $\mathrm{p} 16^{+}$HNSCC, including one patient who developed metastatic disease followed by a complete, rapid and durable response to subseqeunt nivolumab, in a phase I/IIa trial ${ }^{237}$. Other ongoing trials include HARE-40, a phase I/II dose-escalation trial designed to determine the safety of an E7-targeting mRNA vaccine delivered in combination with an agonistic anti-CD40 antibody to enhance antigen presentation by dendritic cells (NCT03418480) and a first-in-human phase I/II trial investigating the novel E6/E7-targeting vaccine HB-201, with or without concurrent immune-checkpoint inhibition (NCT04180215 and NCT03669718) (TABLE 7, Supplementary Information). The results of these trials and others will be crucial in shaping the continued progress of immunotherapies for patients with $\mathrm{HPV}^{+}$ OPSCCs.

\section{Future directions for novel therapies}

Ultimately, with the currently available therapies, identifying safe methods of de-escalation of chemoradiation seems to be a more relevant research objective than identifying the most effective chemotherapy regimen. However, the vast majority of molecular data from patients with $\mathrm{HPV}^{+}$OPSCCs have thus far been derived from primary tumours, $>80 \%$ of which are typically eliminated with chemoradiation. Key challenges are to identify the $15-20 \%$ of primary tumours that are associated with a high risk of recurrence and to identify effective treatments for patients with recurrent disease, for whom 2-year OS remains at $40 \%{ }^{238}$. To this end, sequencing of tumour specimens from 51 patients with primary $\mathrm{HPV}^{+}$OPSCCs, 16 of whom had disease recurrence, together with specimens from 12 metachronous recurrent $\mathrm{HPV}^{+}$OPSCCs (including seven with matched primary tumours also sequenced) was undertaken. This study provided the intriguing observation that recurrent tumours share certain genomic aberrations, such as TP53 mutations, that are almost exclusive to primary $\mathrm{HPV}^{-} \mathrm{HNSCCs}^{239}$. The more recent discovery of a gene-expression profile associated with poor prognosis in patients with $\mathrm{HPV}^{+}$OPSCC that bears similarities to that of $\mathrm{HPV}^{-} \mathrm{HNSCC}$ provides support for this finding. Interestingly, the extent of HPV E6 and E7 expression does not seem to vary between good-prognosis and poor-prognosis $\mathrm{HPV}^{+}$subgroups; instead expression of the viral E1-E4 transcript, which functions during the later stages of the productive HPV replication cycle but has not previously been implicated in cancer, is substantially increased in tumours belonging to the good-prognosis subgroup. The reasons for this increased expression remain unclear but might confer increased radiosensitivity in cells expressing E1-E4 $\left(\mathrm{REF}^{240}\right)$. Given the findings from these studies, determining whether cells derived from recurrent $\mathrm{HPV}^{+}$OPSCCs have the same dependence upon ongoing viral oncogene expression as those derived from primary tumours will be important; if not, this might have implications for the efficacy of HPV-targeted therapies (such as therapeutic vaccines) in patients with advanced-stage disease.

\section{Table 7 | Ongoing immunotherapy clinical trials for $\mathrm{HPV}^{+}$OPSCC}

\begin{tabular}{|c|c|c|c|c|}
\hline Study & Cohort & Treatment & Outcome measures & $\begin{array}{l}\text { Current } \\
\text { status }\end{array}$ \\
\hline $\begin{array}{l}\text { IMvoke010 } \\
\text { (NCT03452137) }\end{array}$ & $\begin{array}{l}406 \text { patients, with a CR/PR or stable } \\
\text { disease following definitive local } \\
\text { therapy }\end{array}$ & $\begin{array}{l}\text { Atezolizumab or placebo as adjuvant } \\
\text { therapy after definitve local therapy } \\
\text { for patients with high-risk disease }\end{array}$ & $\begin{array}{l}\text { EFS (primary outcome), OS } \\
\text { and AEs included as secondary } \\
\text { outcomes }\end{array}$ & Active \\
\hline NCT03799445 & $\begin{array}{l}180 \text { patients with T1 N2a-N2 cM0, T2 } \\
\text { N1-2c M0, T3 N0-2 cM0 (AJCC 7th edn) } \\
\text { or stage I/II disease excluding T1 N0-1 } \\
\text { and T2 N0 (AJCC 8th edn) }\end{array}$ & $\begin{array}{l}\text { IMRT (50-66 Gy) plus nivolumab and } \\
\text { ipilimumab }\end{array}$ & $\begin{array}{l}\text { Dose-limiting toxicities, CR rate, } \\
\text { PFS (primary outcomes); grade } 3 \\
\text { AEs, tolerability, clinical CR, acute } \\
\text { and chronic AEs, acute toxicities, } \\
\text { late toxicities, swallowing, } \\
\text { pattern of failure, OS }\end{array}$ & Recruiting \\
\hline NCT03410615 & $\begin{array}{l}180 \text { patients with locoregionally } \\
\text { advanced, intermediate-risk } \\
\text { non-metastatic disease (AJCC 8th edn) }\end{array}$ & $\begin{array}{l}70 \text { Gy RT with cisplatin vs durvalumab } \\
\text { plus adjuvant durvaluamb } \\
\text { vs durvalumab plus adjuvant } \\
\text { durvalumab/tremelimumab (third arm } \\
\text { closed to accrual) }\end{array}$ & $\begin{array}{l}\text { 3-year EFS (primary outcome); } \\
\text { FACT-HN score, local regional } \\
\text { failure, distant MFS, OS, } \\
\text { cost-effectiveness, toxicities }\end{array}$ & Recruiting \\
\hline NCT03811015 & $\begin{array}{l}744 \text { patients with a } \geq 10 \text { pack-year } \\
\text { smoking history and stage T1-2 N2-3 } \\
\text { or T3-4 N0-3 disease or }<10 \text { pack-years } \\
\text { with stage } \mathrm{T} 4 \mathrm{~N} 0-3 \text { or } \mathrm{T} 1-2 \mathrm{~N} 2-3\end{array}$ & $\begin{array}{l}\text { Cisplatin plus IMRT followed by } \\
\text { nivolumab vs cisplatin plus IMRT } \\
\text { followed by observation with potential } \\
\text { crossover to nivolumab at } 12 \text { months }\end{array}$ & $\begin{array}{l}\text { PFS, OS, negative FDG-PET at } 12 \\
\text { weeks post-therapy }\end{array}$ & Recruiting \\
\hline
\end{tabular}

AJCC, American Joint Committee on Cancer; AEs, adverse events; CR, complete response; DOR, duration of response; EFS, event-free survival; FDG,

fluorodeoxyglucose; FACT-HN, functional assessment of cancer therapy, head \& neck; IMRT, intensity-modulated radiotherapy; MFS, metastasis-free survival; ORR, objective response rate; OS, overall survival; PFS, progression-free survival; PR, partial response; QOL, quality of life; RT, radiotherapy. 
Finally, in the largest genomic analysis of distant metastases from patients with $\mathrm{HPV}^{+}$OPSCC published to date, targeted cancer gene sequencing of samples from 26 metastatic lesions revealed a higher frequency of $P R K D C$ mutations compared with primary tumours. PRKDC encodes the DNA-dependent protein kinase catalytic subunit (DNA-PKcs), which is essential for the repair of DNA double-strand breaks by non-homologous end joining; thus, the investigators speculate that these metastatic tumours might respond to therapies such as PARP inhibitors, which exploit DNA repair defects ${ }^{109}$. Indeed, the PARP inhibitor olaparib is currently being assessed as a radiosensitizer with the aim of improved logoregional control in patients with stage II-III HNSCCs (NCT02229656). However, determining whether these tantalizing observations hold true in larger cohorts of patients with recurrent and/or metastatic $\mathrm{HPV}^{+}$OPSCCs, and developing preclinical models that are representative of these tumours, will be important steps going forward.

\section{Conclusions}

The differentiation of $\mathrm{HPV}^{+}$OPSCCs from their $\mathrm{HPV}^{-}$ counterparts outlined in the 8 th edition of the AJCC staging system cements the distinct biology of these tumours and the associated improved prognosis. The prevalence of these tumours in younger individuals emphasizes the need for continued efforts to treat patients, such that post-treatment QOL remains high. Owing to the particular vulnerability of these tumours to antitumour immunity, novel therapeutic regimens that improve on the associated morbidities and mortality associated with the current standard of care will probably eventually include immunotherapies. Data from completed and ongoing clinical trials emphasize the potential for treatment de-intensification as a means of improving QOL while maintaining robust survival outcomes. More trials are clearly needed, although it remains apparent that such strategies can lead to considerable reductions in morbidity and mortality, and as such, all eligible patients should be considered for such studies. Importantly, a need continues to exist for further research to identify and validate diagnostic, prognostic and predictive biomarkers to improve early detection, stratify patients for potential treatment de-intensification or otherwise better allocate to current standard-of-care therapies and, in future, targeted therapies and immunotherapies.

Published online 1 February 2022
1. Chaturvedi, A. K. et al. Human papillomavirus and rising oropharyngeal cancer incidence in the United States. J. Clin. Oncol. 29, 4294-4301 (2011).

2. Gillison, M. L., Chaturvedi, A. K., Anderson, W. F. \& Fakhry, C. Epidemiology of human papillomavirus positive head and neck squamous cell carcinoma. J. Clin. Oncol. 33, 3235-3242 (2015).

3. Senkomago, V. et al. Human papillomavirus-attributable cancers - United States, 2012-2016. MMWR Morb. Mortal. Wkly Rep. 68, 724-728 (2019)

4. Schache, A. G. et al. HPV-related oropharynx cancer in the United Kingdom: an evolution in the understanding of disease etiology. Cancer Res. 76, 6598-6606 (2016).

5. Lei, J. et al. HPV vaccination and the risk of invasive cervical cancer. N. Engl. J. Med. 383, 1340-1348 (2020).

6. Craig, S. G. et al. Recommendations for determining HPV status in patients with oropharyngeal cancers under TNM8 guidelines: a two-tier approach. $\mathrm{Br}$. J. Cancer 120, 827-833 (2019).

7. Gillison, M. L. et al. Distinct risk factor profiles for human papillomavirus type 16-positive and human papillomavirus type 16-negative head and neck cancers. J. Natl Cancer Inst. 100, 407-420 (2008).

8. Lechner, M., Jones, O. S., Breeze, C. E. \& Gilson, R. Gender-neutral HPV vaccination in the UK, rising male oropharyngeal cancer rates, and lack of HPV awareness. Lancet Infect. Dis. 19, 131-132 (2019).

9. Faraji, F. et al. The prevalence of human papillomavirus in oropharyngeal cancer is increasing regardless of sex or race, and the influence of sex and race on survival is modified by human papillomavirus tumor status. Cancer 125, 761-769 (2019).

10. Argirion, I. et al. Increasing prevalence of HPV in oropharyngeal carcinoma suggests adaptation of p16 screening in Southeast Asia. J. Clin. Virol. 132 104637 (2020).

11. Hwang, T. Z., Hsiao, J. R., Tsai, C. R. \& Chang, J. S Incidence trends of human papillomavirus-related head and neck cancer in Taiwan, 1995-2009. Int. J. Cancer 137, 395-408 (2015).

12. Wittekindt, C. et al. Increasing incidence rates of oropharyngeal squamous cell carcinoma in Germany and significance of disease burden attributed to human papillomavirus. Cancer Prev. Res. 12 375-382 (2019)

13. Zamani, M. et al. The current epidemic of HPV-associated oropharyngeal cancer: an 18-year Danish population-based study with 2,169 patients Eur. J. Cancer 134, 52-59 (2020).
14. Del Mistro, A. et al. Age-independent increasing prevalence of human papillomavirus-driven oropharyngeal carcinomas in North-East Italy. Sci. Rep. 10, 1-10 (2020)

15. Morbini, P. et al. The evolving landscape of human papillomavirus-related oropharyngeal squamous cell carcinoma at a single institution in northern Italy. Acta Otorhinolaryngol. Ital. 39, 9-17 (2019).

16. Haeggblom, L. et al. Changes in incidence and prevalence of human papillomavirus in tonsillar and base of tongue cancer during 2000-2016 in the Stockholm region and Sweden. Head. Neck 41 , 1583-1590 (2019).

17. Donà, M. G. et al. Evolving profile of HPV-driven oropharyngeal squamous cell carcinoma in a national cancer institute in Italy: a 10-year retrospective study. Microorganisms 8, 1-12 (2020).

18. Girardi, F. M., Wagner, V. P., Martins, M. D., Abentroth, A. L. \& Hauth, L. A. Prevalence of p16 expression in oropharyngeal squamous cell carcinoma in southern Brazil. Oral. Surg. Oral. Med. Oral. Pathol. Oral. Radiol. 130, 681-691 (2020).

19. Rietbergen, M. M. et al. Epidemiologic associations of HPV-positive oropharyngeal cancer and (pre) cancerous cervical lesions. Int. J. Cancer 143 283-288 (2018)

20. Carlander, A. F. et al. A contemporary systematic review on repartition of HPV-positivity in oropharyngeal cancer worldwide. Viruses 13, 1326 (2021)

21. Chen, S. Y. et al. The association of smoking and outcomes in HPV-positive oropharyngeal cancer: a systematic review. Am. J. Otolaryngol. 41, 102592 (2020).

22. Ang, K. K. et al. Human papillomavirus and survival of patients with oropharyngeal cancer. N. Engl. J. Med. 363, 24-35 (2010).

23. Gooi, Z Chan, J Y K K \& Fakhry C. The epidemiology of the human papillomavirus related to oropharyngeal head and neck cancer. Laryngoscope 126, 894-900 (2016).

24. D'Souza, G. et al. Sex differences in risk factors and natural history of oral human papillomavirus infection. J. Infect. Dis. 213, 1893-1896 (2016).

25. de Martel, C., Plummer, M., Vignat, J. \& Franceschi, S. Worldwide burden of cancer attributable to HPV by site, country and HPV type. Int. J. Cancer 141 , 664-670 (2017)

26. Blumberg, J., Monjane, L., Prasad, M., Carrilho, C. $\&$ Judson, B. L. Investigation of the presence of HPV related oropharyngeal and oral tongue squamous cell carcinoma in Mozambique. Cancer Epidemiol. 39 1000-1005 (2015).
27. Rettig, E. M. et al. Oral human papillomavirus infection and head and neck squamous cell carcinoma in rural northwest Cameroon. OTO Open 3 , $2473974 X 18818415$ (2019)

28. Ndiaye, C. et al. The role of human papillomavirus in head and neck cancer in Senegal. Infect. Agent. Cancer 8, 14 (2013).

29. Kofi, B. et al. Infrequent detection of human papillomavirus infection in head and neck cancers in the Central African Republic: a retrospective study. Infect. Agent Cancer 14, 9 (2019).

30. Oga, E. A. et al. Paucity of HPV-related head and neck cancers (HNC) in Nigeria. PLoS ONE 11, e0152828 (2016).

31. Chaturvedi, A. K. \& Zumsteg, Z. S. A snapshot of the evolving epidemiology of oropharynx. cancers Cancer 124, 2893-2896 (2018).

32. Tota, J. E. et al. Evolution of the oropharynx cancer epidemic in the United States: moderation of increasing incidence in younger individuals and shift in the burden to older individuals. J. Clin. Oncol. 37. 1538-1546 (2019).

33. Kreimer, A. R. et al. Summary from an international cancer seminar focused on human papillomavirus (HPV)-positive oropharynx cancer, convened by scientists at IARC and NCI. Oral. Oncol. 108, 104736 (2020).

34. Mariz BALA, K. L. P. et al. Global prevalence of human papillomavirus-driven oropharyngeal squamous cell carcinoma following the ASCO guidelines: a systematic review and meta-analysis. Crit. Rev. Oncol. Hematol. 156, 103116 (2020)

35. Windon, M. J. et al. Increasing prevalence of human papillomavirus-positive oropharyngeal cancers among older adults. Cancer 124, 2993-2999 (2018).

36. Rettig, E. M., Fakhry, C., Khararjian, A. \& Westra, W. H. Age profile of patients with oropharyngeal squamous cell carcinoma. JAMA Otolaryngol. 144, 538-539 (2018).

37. Zumsteg, Z. S. et al. Incidence of oropharyngeal cancer among elderly patients in the United States. JAMA Oncol. 2, 1617-1623 (2016).

38. Mahal, B. A. et al. Incidence and demographic burden of HPV-associated oropharyngeal head and neck cancers in the United States. Cancer Epidemiol. Biomark. Prev. 28, 1660-1667 (2019).

39. Ramer, l. et al. Racial disparities in incidence of human papillomavirus-associated oropharyngeal cancer in an urban population. Cancer Epidemiol. 44, 91-95 (2016). 
40. Liederbach, E. et al. The national landscape of human papillomavirus-associated oropharynx squamous cell carcinoma. Int. J. Cancer 140, 504-512 (2017).

41. Falcaro, M. et al. The effects of the national HPV vaccination programme in England, UK, on cervical cancer and grade 3 cervical intraepithelial neoplasia incidence: a register-based observational study. Lancet 398, 2084-2092 (2021).

42. Masterson, L. \& Lechner, M. HPV vaccination in boys - will the UK join the fight? Nat. Rev. Clin. Oncol. 13, 721-722 (2016).

43. HPV Vaccination Uptake. Australia National Control Indicators. Published 2019. https:// ncci.canceraustralia.gov.au/prevention/hpv vaccination-uptake/hpv-vaccination-uptake (2021)

44. Walker, T. Y. et al. National, regional, state, and selected local area vaccination coverage among adolescents aged 13-17 years - United States, 2019. MMWR Morb. Mortal. Wkly Rep. 69, 1109-1116 (2020).

45. Public Health England. Human papillomavirus (HPV) vaccination coverage in adolescent females and males in England: academic year 2019 to 2020. Heal. Prot. Rep. 14, 1-15 (2020)

46. Radisic, G., Chapman, J., Flight, I. \& Wilson, C. Factors associated with parents' attitudes to the HPV vaccination of their adolescent sons: a systematic review. Prev. Med. 95, 26-37 (2017).

47. Sonawane, K. et al. Parental intent to initiate and complete the human papillomavirus vaccine series in the USA: a nationwide, cross-sectional survey. Lancet Public Heal. 5, e484-e492 (2020).

48. Gottvall, M., Stenhammar, C. \& Grandahl, M. Parents' views of including young boys in the Swedish national school-based HPV vaccination programme: a qualitative study. BMJ Open 7, 11-13 (2017).

49. Thompson, E. L. et al. Awareness and knowledge of HPV and HPV vaccination among adults ages 27-45 years. Vaccine 38, 3143-3148 (2020)

50. Waller, J. et al. Decision-making about HPV vaccination in parents of boys and girls: a population-based survey in England and Wales. Vaccine 38, 1040-1047 (2020).

51. Sherman, S. M., Cohen, C. R., Denison, H. J., Bromhead, C. \& Patel, H. A survey of knowledge, attitudes and awareness of the human papillomavirus among healthcare professionals across the UK. Eur. J. Public Health 30, 10-16 (2020).

52. Lechner, M. et al. A cross-sectional survey of awareness of human papillomavirus-associated oropharyngeal cancers among general practitioners in the UK. BMJ Open 8, 1-6 (2018).

53. Katz, J. The impact of HPV vaccination on the prevalence of oropharyngeal cancer (OPC) in a hospital-based population: a cross-sectional study of patient's registry. J. Oral. Pathol. Med. 50, 47-51 (2021).

54. Herrero, R. et al. Reduced prevalence of oral human papillomavirus (HPV) 4 years after bivalent HPV vaccination in a randomized clinical trial in Costa Rica. PLOS ONE 8, e68329 (2013).

55. Chaturvedi, A. K. et al. Effect of prophylactic human papillomavirus (HPV) vaccination on oral HPV infections among young adults in the United States. J. Clin. Oncol. 36, 262-267 (2018).

56. Hirth, J. M., Chang, M., Resto, V. A., Guo, F. \& Berenson, A. B. Prevalence of oral human papillomavirus by vaccination status among young adults (18-30 years old). Vaccine $35,3446-3451$ (2017).

57. Zhang, Y., Fakhry, C. \& D'Souza, G. Projected association of human papillomavirus vaccination with oropharynx cancer incidence in the US, 2020-2045. JAMA Oncol. 7, e212907 (2021).

58. Lechner, M., Breeze, C. E., O'Mahony, J. F. \& Masterson, L. Early detection of HPV-associated oropharyngeal cancer. Lancet 393, 2123 (2019).

59. Kreimer, A. R. et al. Timing of HPV16-E6 antibody seroconversion before OPSCC: findings from the HPVC3 consortium. Ann. Oncol. 30, 1335-1343 (2019).

60. Kreimer, A. R., Clifford, G. M., Boyle, P. \& Franceschi, S. Human papillomavirus types in head and neck squamous cell carcinomas worldwide: a systemic review. Cancer Epidemiol. Biomark. Prev. 14 467-475 (2005)

61. Egawa, N., Egawa, K., Griffin, H. \& Doorbar, J. Human papillomaviruses; epithelial tropisms, and the development of neoplasia. Viruses 7, 3863-3890 (2015).

62. Doorbar, J. et al. The biology and life-cycle of human papillomaviruses. Vaccine 30, F55-F70 (2012).
63. Graham, S. V. Keratinocyte differentiation-dependent human papillomavirus gene regulation. Viruses 9 245 (2017).

64. Parfenov, M. et al. Characterization of HPV and host genome interactions in primary head and neck cancers. Proc. Natl Acad. Sci. USA 111 15544-15549 (2014).

65. Vinokurova, S. et al. Type-dependent integration frequency of human papillomavirus genomes in cervical lesions. Cancer Res. 68, 307-313 (2008).

66. Ramqvist, T. et al. Studies on human papillomavirus (HPV) 16 E2, E5 and E7 mRNA in HPV-positive tonsillar and base of tongue cancer in relation to clinical outcome and immunological parameters. Oral. Oncol. 51, 1126-1131 (2015).

67. Koneva, L. A. et al. HPV integration in HNSCC correlates with survival outcomes, immune response signatures, and candidate drivers. Mol. Cancer Res. 16, 90-102 (2018)

68. Hanahan, D. \& Weinberg, R. A. Hallmarks of cancer: the next generation. Cell 144, 646-674 (2011).

69. Mesri, E. A., Feitelson, M. A. \& Munger, K. Human viral oncogenesis: a cancer hallmarks analysis. Cell Host Microbe 15, 266-282 (2014).

70. Huibregtse, J. M., Scheffner, M. \& Howley, P. M. A cellular protein mediates association of p53 with the E6 oncoprotein of human papillomavirus types 16 or 18. EMBO J. 10, 4129-4135 (1991)

71. Scheffner, M., Werness, B. A., Huibregtse, J. M. Levine, A. J. \& Howley, P. M. The E6 oncoprotein encoded by human papillomavirus types 16 and 18 promotes the degradation of p53. Cell 63 , 1129-1136 (1990)

72. Scheffner, M., Huibregtse, J. M., Vierstra, R. D. \& Howley, P. M. The HPV-16 E6 and E6-AP complex functions as a ubiquitin-protein ligase in the ubiquitination of p53. Cell 75, 495-505 (1993).

73. Huh, K. et al. Human papillomavirus type 16 E7 oncoprotein associates with the Cullin 2 ubiquitin ligase complex, which contributes to degradation of the retinoblastoma tumor suppressor. J. Virol. $\mathbf{8 1}$, 9737-9747 (2007).

74. Dyson, N., Howley, P. M., Munger, K. \& Harlow, E. The human papilloma virus-16 E7 oncoprotein is able to bind to the retinoblastoma gene product. Science 243, 934-938 (1986)

75. Münger, K., Phelps, W. C., Bubb, V., Howley, P. M $\&$ Schlegel, R. The E6 and E7 genes of the human papillomavirus type 16 together are necessary and sufficient for transformation of primary human keratinocytes. J. Virol. 63, 4417-4421 (1989).

76. Božinović, K. et al. Genome-wide miRNA profiling reinforces the importance of miR-9 in human papillomavirus associated oral and oropharyngeal head and neck cancer. Sci. Rep. 9, 2306 (2019).

77. Boscolo-Rizzo, P., Furlan, C., Lupato, V., Polesel, J. \& Fratta, E. Novel insights into epigenetic drivers of oropharyngeal squamous cell carcinoma: role of HPV and lifestyle factors. Clin. Epigenetics 9, 124 (2017).

78. Barr, J. A. et al. Long non-coding RNA FAM83H-AS1 is regulated by human papillomavirus $16 \mathrm{E} 6$ independently of p53 in cervical cancer cells. Sci. Rep. 9, 3662 (2019).

79. Lechner, M. et al. Identification and functional validation of HPV-mediated hypermethylation in head and neck squamous cell carcinoma. Genome Med. $\mathbf{5}$, 15 (2013)

80. Burgers, W. A. et al. Viral oncoproteins target the DNA methyltransferases. Oncogene 26, 1650-1655 (2007).

81. Chalertpet, K., Pakdeechaidan, W., Patel, V., Mutirangura, A. \& Yanatatsaneejit, P. Human papillomavirus type 16 E7 oncoprotein mediates CCNA1 promoter methylation. Cancer Sci. 106, 1333-1340 (2015).

82. Cicchini, L. et al. Suppression of antitumor immune responses by human papillomavirus through epigenetic downregulation of CXCL14. mBio 7 e00270-16 (2016)

83. Cicchini, L. et al. High-risk human papillomavirus E7 alters host DNA methylome and represses HLA-E expression in human keratinocytes. Sci. Rep. 7, 3633 (2017).

84. Munger, K. \& Jones, D. L. Human papillomavirus carcinogenesis: an identity crisis in the retinoblastoma tumor suppressor pathway. J. Virol. 89, 4708-4711 (2015).

85. Magaldi, T. G. et al. Primary human cervical carcinoma cells require human papillomavirus E6 and E7 expression for ongoing proliferation. Virology 422 , 114-124 (2012)
86. Weinstein, I. B. Addiction to oncogenes-the Achilles heal of cancer. Science 297, 63-64 (2002).

87. McLaughlin-Drubin, M. E., Crum, C. P. \& Münger, K Human papillomavirus E7 oncoprotein induces KDM6A and KDM6B histone demethylase expression and causes epigenetic reprogramming. Proc. Natl Acad. Sci. USA 108, 2130-2135 (2011).

88. McLaughlin-Drubin, M. E., Park, D. \& Munger, K. Tumor suppressor p16INK4A is necessary for survival of cervical carcinoma cell lines. Proc. Natl Acad. Sci. USA 110, 16175-16180 (2013).

89. Spring, L. M. et al. Cyclin-dependent kinase 4 and 6 inhibitors for hormone receptor-positive breast cancer: past, present, and future. Lancet 395 , 817-827 (2020).

90. Soto, D. R., Barton, C., Munger, K. \&

McLaughlin-Drubin, M. E. KDM6A addiction of cervical carcinoma cell lines is triggered by E7 and mediated by $\mathrm{p} 21^{\mathrm{CIP} 1}$ suppression of replication stress. PLoS Pathog. 13, 1-25 (2017).

91. Ganti, K. et al. The human papillomavirus E6 PDZ binding motif: from life cycle to malignancy. Viruses 7 3530-3551 (2015)

92. Mittal, S. \& Banks, L. Molecular mechanisms underlying human papillomavirus E6 and E7 oncoprotein-induced cell transformation. Mutat. Res. 772, 23-35 (2017).

93. Roman, A. \& Munger, K. The papillomavirus E7 proteins. Virology 445, 138-168 (2013).

94. Moody, C. A. \& Laimins, L. A. Human papillomavirus oncoproteins: pathways to transformation. Nat. Rev. Cancer 10, 550-560 (2010).

95. Seiwert, T. Y. et al. Integrative and comparative genomic analysis of HPV-positive and HPV-negative head and neck squamous cell carcinomas. Clin. Cancer Res. 21, 632-641 (2015).

96. The Cancer Genome Atlas Network. Comprehensive genomic characterization of head and neck squamous cell carcinomas. Nature 517, 576-582 (2015)

97. Gillison, M. L. et al. Human papillomavirus and the landscape of secondary genetic alterations in oral cancers. Genome Res. 29, 1-17 (2019).

98. Dogan, S. et al. Identification of prognostic molecular biomarkers in $157 \mathrm{HPV}$-positive and HPV-negative squamous cell carcinomas of the oropharynx. Int. $J$. Cancer 145, 3152-3162 (2019).

99. Lechner, M. et al. Targeted next-generation sequencing of head and neck squamous cell carcinoma identifies novel genetic alterations in HPV and HPV-tumors. Genome Med. 5, 49 (2013).

100. Hayes, D. N., Van Waes, C. \& Seiwert, T. Y. Genetic landscape of human papillomavirus-associated head and neck cancer and comparison to tobacco-related tumors. J. Clin. Oncol. 33, 3227-3234 (2015).

101. Henderson, S., Chakravarthy, A., Su, X., Boshoff, C. \& Fenton, T. R. APOBEC-mediated cytosine deamination links PIK3CA helical domain mutations to human papillomavirus-driven tumor development. Cell Rep. 7, 1833-1841 (2014).

102. Zhu, B. et al. Mutations in the HPV16 genome induced by APOBEC3 are associated with viral clearance. Nat. Commun. 11, 1-12 (2020).

103. Faden, D. L. et al. APOBEC mutagenesis is concordant between tumor and viral genomes in HPV-positive head and neck squamous cell carcinoma. Viruses 13 1666 (2021).

104. Smith, N. J. \& Fenton, T. R. The APOBEC3 genes and their role in cancer: insights from human papillomavirus. J. Mol. Endocrinol. 62, R269-R287 (2019)

105. Fenton, T. R. Accumulation of host cell genetic errors following high-risk HPV infection. Curr. Opin. Virol. $\mathbf{5 1}$ 1-8 (2021).

106. Warren, C. J., Westrich, J. A., Van Doorslaer, K. \& Pyeon, D. Roles of APOBEC3A and APOBEC3B in human papillomavirus infection and disease progression. Viruses 9, 1-20 (2017).

107. Lui, V. W. Y. et al. Frequent mutation of the PI3K pathway in head and neck cancer defines predictive biomarkers. Cancer Discov. 3, 761-769 (2013).

108. Nichols, A. C. et al. High frequency of activating PIK3CA mutations in human papillomavirus- positive oropharyngeal cancer. JAMA Otolaryngol. 139, 617-622 (2013)

109. Hanna, G. J. et al. Improved outcomes in PI3K-pathway-altered metastatic HPV oropharyngeal cancer. JCl Insight 3, e122799 (2018).

110. Beaty, B. T. et al. PIK3CA mutation in HPV associated OPSCC patients receiving deintensified chemoradiation. J. Natl Cancer Inst. 112, 855-858 (2019). 
111. Hedberg, M. L. et al. Use of nonsteroidal anti-inflammatory drugs predicts improved patient survival for PIK3CA-altered head and neck cancer. J. Exp. Med. 216, 419-427 (2019)

112. Cai, Y., Yousef, A., Grandis, J. R. \& Johnson, D. E. NSAID therapy for PIK3CA-altered colorectal, breast, and head and neck cancer. Adv. Biol. Regul. 75 100653 (2020)

113. Paleari, L. et al. PIK3CA mutation, aspirin use after diagnosis and survival of colorectal cancer. a systematic review and meta-analysis of epidemiological studies. Clin. Oncol. 28, 317-326 (2016).

114. Nyman, P. E., Buehler, D. \& Lambert, P. F. Loss of function of canonical Notch signaling drives head and neck carcinogenesis. Clin. Cancer Res. 24 , 6308-6318 (2018)

115. Kranjec, C. et al. Modulation of basal cell fate during productive and transforming HPV-16 infection is mediated by progressive E6-driven depletion of Notch. J. Pathol. 242, 448-462 (2017).

116. Beglin, M., Melar-New, M. \& Laimins, L. Human papillomaviruses and the interferon response. J. Interf. Cytokine Res. 29, 629-635 (2009).

117. Dhawan, A. et al. Role of gene signatures combined with pathology in classification of oropharynx head and neck cancer. Sci. Rep. 10, 10226 (2020).

118. She, Y. et al. Immune-related gene signature for predicting the prognosis of head and neck squamous cell carcinoma. Cancer Cell Int. 20, 22 (2020).

119. Chan, T. A. et al. Development of tumor mutation burden as an immunotherapy biomarker: utility for the oncology clinic. Ann. Oncol. 30, 44-56 (2019).

120. McGranahan, N. et al. Clonal neoantigens elicit $\mathrm{T}$ cell immunoreactivity and sensitivity to immune checkpoint blockade. Science 351, 1463-1469 (2016).

121. Schreiber, R. D., Old, L. J. \& Smyth, M. J. Cancer immunoediting: integrating immunity's roles in cancer suppression and promotion. Science 331 , 1565-1570 (2011)

122. Steinbach, A. \& Riemer, A. B. Immune evasion mechanisms of human papillomavirus: an update. Int. J. Cancer 142, 224-229 (2018).

123. Ashrafi, G. H., Haghshenas, M. R., Marchetti, B., O'Brien, P. M. \& Campo, M. S. E5 protein of human papillomavirus type 16 selectively downregulates surface HLA class I. Int. J. Cancer 113, 276-283 (2005).

124. Ashrafi, G. H., Haghshenas, M., Marchetti, B. \& Campo, M. S. E5 protein of human papillomavirus 16 downregulates HLA class I and interacts with the heavy chain via its first hydrophobic domain. Int. $J$. Cancer 119, 2105-2112 (2006).

125. Campo, M. S. et al. HPV-16 E5 down-regulates expression of surface HLA class I and reduces recognition by CD8 T cells. Virology 407, 137-142 (2010)

126. Georgopoulos, N. T., Proffitt, J. L. \& Blair, G. E. Transcriptional regulation of the major histocompatibility complex (MHC) class I heavy chain, TAP1 and LMP2 genes by the human papillomavirus (HPV) type 6b, 16 and 18 E7 oncoproteins. Oncogene 19, 4930-4935 (2000).

127. Li, H., Ou, X., Xiong, J. \& Wang, T. HPV16E7 mediates HADC chromatin repression and downregulation of MHC class I genes in HPV16 tumorigenic cells through interaction with an MHC class I promoter. Biochem. Biophys. Res. Commun. 349, 1315-1321 (2006).

128. Bottley, G. et al. High-risk human papillomavirus E7 expression reduces cell-surface MHC class I molecules and increases susceptibility to natural killer cells. Oncogene 27, 1794-1799 (2008).

129. Heusinkveld, M. et al. Systemic and local human papillomavirus 16-specific T-cell immunity in patients with head and neck cancer. Int. J. Cancer 131, 74-85 (2012).

130. Welters, M. J. P. et al. Intratumoral HPV16 specific $\mathrm{T}$ cells constitute a type l-oriented tumor microenvironment to improve survival in HPV16driven oropharyngeal cancer. Clin. Cancer Res. 24 , 634-647 (2018).

131. Santegoets, S. J. et al. The anatomical location shapes the immune infiltrate in tumors of same etiology and affects survival. Clin. Cancer Res. 25, 240-252 (2019).

132. Santegoets, S. J. et al. CD $163^{+}$cytokine-producing CDC2 stimulate intratumoral type $1 \mathrm{~T}$ cell responses in HPV16-induced oropharyngeal cancer. J. Immunother Cancer 8, e001053 (2020)

133. Hoffmann, T. K. et al. T cells specific for HPV 16 E7 epitopes in patients with squamous cell carcinoma of the oropharynx. Int. J. Cancer 118, 1984-1991 (2006).

134. Masterson, L. et al. CD8+ T cell response to human papillomavirus $16 \mathrm{E7}$ is able to predict survival outcome in oropharyngeal cancer. Eur. J. Cancer 67 141-151 (2016)

135. Laban, S. \& Hoffmann, T. K. Human papillomavirus immunity in oropharyngeal cancer: time to change the game? Clin. Cancer Res. 24, 505-507 (2018).

136. Balermpas, P. et al. CD8+ tumour-infiltrating lymphocytes in relation to HPV status and clinical outcome in patients with head and neck cancer after postoperative chemoradiotherapy: a multicentre study of the German Cancer Consortium Radiation Oncology Group (DKTK-ROG). Int. J. Cancer 138, 171-181 (2016).

137. Ward, M. J. et al. Tumour-infiltrating lymphocytes predict for outcome in HPV-positive oropharyngeal cancer. Br. J. Cancer 110, 489-500 (2014).

138. Mandal, R. et al. The head and neck cancer immune landscape and its immunotherapeutic implications. JCl Insight 1, e89829 (2016).

139. Chakravarthy, A. et al. Human papillomavirus drives tumor development throughout the head and neck: improved prognosis is associated with an immune response largely restricted to the oropharynx. J. Clin. Oncol. 34, 4132-4141 (2016)

140. Li, H. et al. Association of human papillomavirus status at head and neck carcinoma subsites with overall survival. JAMA Otolaryngol. 144, 519-525 (2018).

141. Hladíková, K. et al. Tumor-infiltrating B cells affect the progression of oropharyngeal squamous cell carcinoma via cell-to-cell interactions with CD8 T cells. J. Immunother. Cancer 7, 261 (2019).

142. Wood, O. et al. Gene expression analysis of TIL rich HPV-driven head and neck tumors reveals a distinct B-cell signature when compared to HPV independent tumors. Oncotarget 7, 56781-56797 (2016).

143. Ou, D. et al. Influence of tumor-associated macrophages and HLA class I expression according to HPV status in head and neck cancer patients receiving chemo/bioradiotherapy. Radiother. Oncol. 130 89-96 (2019).

144. Welters, M. J. P., Santegoets, S. J. \& van der Burg, S. H. The tumor microenvironment and immunotherapy of oropharyngeal squamous cell carcinoma. Front. Oncol. 10, 545385 (2020).

145. Hong, A. M. et al. Significant association of PD-L1 expression with human papillomavirus positivity and its prognostic impact in oropharyngeal cancer. Oral. Oncol. 92, 33-39 (2019).

146. Cao, S. et al. Dynamic host immune response in virus-associated cancers. Commun. Biol. 2, 109 (2019).

147. Mcllwain, W. R., Sood, A. J., Nguyen, S. A. \& Day, T. A. Initial symptoms in patients with HPV-positive and HPV-negative oropharyngeal cancer. JAMA Otolaryngol. 140, 441-447 (2014).

148. Khalid, M. B. et al. Initial presentation of human papillomavirus-related head and neck cancer: a retrospective review. Laryngoscope 129, 877-882 (2019).

149. Tham, T., Ahn, S., Frank, D., Kraus, D. \& Costantino, P. Anatomical subsite modifies survival in oropharyngeal squamous cell carcinoma: National Cancer Database study. Head. Neck 42, 434-445 (2020).

150. Golusinski, P. et al. Evidence for the approach to the diagnostic evaluation of squamous cell carcinoma occult primary tumors of the head and neck. Oral. Oncol. 88, 145-152 (2019).

151. Zhang, M. Q., El-Mofty, S. K. \& Dávila, R. M Detection of human papillomavirus-related squamous cell carcinoma cytologically and by in situ hybridization in fine-needle aspiration biopsies of cervical metastasis: a tool for identifying the site of an occult head and neck primary. Cancer 114, 118-123 (2008).

152. Begum, S., Gillison, M. L., Nicol, T. L. \& Westra, W. H. Detection of human papillomavirus-16 in fine-needle aspirates to determine tumor origin in patients with metastatic squamous cell carcinoma of the head and neck. Clin. Cancer Res. 13, 1186-1191 (2007).

153. Mehanna, H. et al. Oropharyngeal cancer: United Kingdom National Multidisciplinary Guidelines. J. Laryngol. Otol. 130, S90-S96 (2016).

154. Gage, K. L., Thomas, K., Jeong, D., Stallworth, D. C. $\&$ Arrington, J. A. Multimodal imaging of head and neck squamous cell carcinoma. Cancer Control. 24 172-179 (2017)
155. Schache, A. G. et al. Evaluation of human papilloma virus diagnostic testing in oropharyngeal squamous cell carcinoma: sensitivity, specificity, and prognostic discrimination. Clin. Cancer Res. 17, 6262-6271 (2011).

156. Lewis, J. S. Morphologic diversity in human papillomavirus-related oropharyngeal squamous cell carcinoma: catch me if you can! Mod. Pathol. 30 S44-S53 (2017)

157. Chernock, R. D., Lewis, J. S., Zhang, Q. \& El-Mofty, S. K. Human papillomavirus-positive basaloid squamous cell carcinomas of the upper aerodigestive tract: a distinct clinicopathologic and molecular subtype of basaloid squamous cell carcinoma. Hum. Pathol. 41, 1016-1023 (2010).

158. Cho, K. J. et al. Basaloid squamous cell carcinoma of the head and neck: subclassification into basal, ductal, and mixed subtypes based on comparison of clinico-pathologic features and expression of p53, cyclin D1, epidermal growth factor receptor, p16, and human papilloma. J. Pathol. Transl. Med. 51 374-380 (2017).

159. Mehrad, M. et al. Papillary squamous cell carcinoma of the head and neck: clinicopathologic and molecular features with special reference to human papillomavirus. Am. J. Surg. Pathol. 37, 1349-1356 (2013).

160. Carpenter, D. H., El-Mofty, S. K. \& Lewis, J. S. Undifferentiated carcinoma of the oropharynx: a human papillomavirus-associated tumor with a favorable prognosis. Mod. Pathol. 24, 1306-1312 (2011).

161. Singhi, A. D., Stelow, E. B., Mills, S. E. \& Westra, W. H. Lymphoepithelial-like carcinoma of the oropharynx: a morphologic variant of HPV-related head and neck carcinoma. Am. J. Surg. Pathol. 34, 800-805 (2010).

162. Jo, V. Y., Mills, S. E., Stoler, M. H. \& Stelow, E. B. Papillary squamous cell carcinoma of the head and neck: frequent association with human papillomavirus infection and invasive carcinoma. Am. J. Surg. Pathol. 33, 1720-1724 (2009).

163. Bryne, M., Koppang, H. S., Lilleng, R. \& Kjærheim, $\AA$ Malignancy grading of the deep invasive margins of oral squamous cell carcinomas has high prognostic value. J. Pathol. 166, 375-381 (1992).

164. Albergotti, W. G. et al. Defining the prevalence and prognostic value of perineural invasion and angiolymphatic invasion in human papillomavirus-positive oropharyngeal carcinoma. JAMA Otolaryngol. 143, 1236-1243 (2017).

165. Dirven, R. et al. Tumor thickness versus depth of invasion - analysis of the 8th edition American Joint Committee on Cancer Staging for oral cancer. Oral. Oncol. 74, 30-33 (2017).

166. Zhan, K. Y. et al. Appraisal of the AJCC 8th edition pathologic staging modifications for HPV-positive oropharyngeal cancer, a study of the National Cancer Data Base. Oral. Oncol. 73, 152-159 (2017)

167. Elicin, O. et al. Comparison of contemporary staging systems for oropharynx cancer in a surgically treated multi-institutional cohort. Head. Neck 41 1395-1402 (2019)

168. Bhattasali, O., Thompson, L. D. R., Schumacher, A. J. \& Iganej, S. Radiographic nodal prognostic factors in stage I HPV-related oropharyngeal squamous cell carcinoma. Head. Neck 41, 398-402 (2019).

169. Sinha, P. et al. High metastatic node number, not extracapsular spread or $\mathrm{N}$-classification is a node-related prognosticator in transorally-resected, neck-dissected p16-positive oropharynx cancer. Oral. Oncol. 51, 514-520 (2015).

170. Bauer, E. et al. Extranodal extension is a strong prognosticator in HPV-positive oropharyngeal squamous cell carcinoma. Laryngoscope 130 939-945 (2020).

171. Freitag, J. et al. Extracapsular extension of neck nodes and absence of human papillomavirus 16-DNA are predictors of impaired survival in p16-positive oropharyngeal squamous cell carcinoma. Cancer 126, 1856-1872 (2020).

172. Tian, S. et al. Prognostic value of radiographically defined extranodal extension in human papillomavirus-associated locally advanced oropharyngeal carcinoma. Head. Neck 41, 3056-3063 (2019).

173. Meyer, M. F. et al. The relevance of the lymph node ratio as predictor of prognosis is higher in HPV-negative than in HPV-positive oropharyngeal squamous cell carcinoma. Clin. Otolaryngol. 43, 92-198 (2018). 
174. Chai, R. L. et al. Accuracy of computed tomography in the prediction of extracapsular spread of lymph node metastases in squamous cell carcinoma of the head and neck. JAMA Otolaryngol. 139, 1187-1194 (2013).

175. Aiken, A. H. et al. Accuracy of preoperative imaging in detecting nodal extracapsular spread in oral cavity squamous cell carcinoma. Am. J. Neuroradiol. 36 1776-1781 (2015)

176. Carlton, J. A. et al. Computed tomography detection of extracapsular spread of squamous cell carcinoma of the head and neck in metastatic cervical lymph nodes. Neuroradiol. J. 30, 222-229 (2017).

177. Douglas, C. et al. Accuracy of contrast-enhanced CT and predictive factors for extracapsular spread in unknown primary head and neck squamous cell cancer. Clin. Radiol. 75, 77.e23-77.e28 (2020).

178. O'Sullivan, B. et al. Development and validation of a staging system for HPV-related oropharyngeal cancer by the International Collaboration on Oropharyngeal cancer Network for Staging (ICON-S): a multicentre cohort study. Lancet Oncol. 17 440-451 (2016).

179. Cramer, J. D., Hicks, K. E., Rademaker, A. W., Patel, U. A. $\&$ Samant, S. Validation of the eighth edition American Joint Committee on Cancer staging system for human papillomavirus-associated oropharyngeal cancer. Head. Neck 40, 457-466 (2018).

180. Geltzeiler, M. et al. Staging HPV-related oropharyngeal cancer: validation of AJCC-8 in a surgical cohort. Oral. Oncol. 84, 82-87 (2018)

181. Van Gysen, K. et al. Validation of the 8th edition UICC/AJCC TNM staging system for HPV associated oropharyngeal cancer patients managed with contemporary chemo-radiotherapy. BMC Cancer 19 674 (2019).

182. Würdemann, N. et al. Prognostic impact of AJCC/UICC 8th edition new staging rules in oropharyngeal squamous cell carcinoma. Front. Oncol. 7, 129 (2017).

183. Nauta, I. H. et al. Evaluation of the eighth TNM classification on p16-positive oropharyngeal squamous cell carcinomas in the Netherlands and the importance of additional HPV DNA testing. Ann. Oncol. 29, 1273-1279 (2018)

184. Fakhry, C. et al. Validation of NRG oncology/RTOG 0129 risk groups for HPV-positive and HPV-negative oropharyngeal squamous cell cancer: implications for risk-based therapeutic intensity trials. Cancer $\mathbf{1 2 5}$ 2027-2038 (2019)

185. Haeggblom, L., Ramqvist, T., Tommasino, M. \& Dalianis, T. Time to change perspectives on HPV in oropharyngeal cancer. A systematic review of HPV prevalence per oropharyngeal sub-site the last 3 years. Papillomavirus Res. 4, 1-11 (2017).

186. Wendt, M. et al. Long-term survival and recurrence in oropharyngeal squamous cell carcinoma in relation to subsites, HPV, and p16-status. Cancers (Basel) 13. 2553 (2021)

187. Ellis, M. et al. Post-treatment head and neck cancer care: national audit and analysis of current practice in the United Kingdom. Clin. Otolaryngol. 46, 284-294 (2021).

188. Fakhry, C et al. Association of oral human papillomavirus DNA persistence with cancer progression after primary treatment for oral cavity and oropharyngeal squamous cell carcinoma. JAMA Oncol. 5, 985-992 (2019).

189. Chera, B. S. et al. Plasma circulating tumor HPV DNA for the surveillance of cancer recurrence in HPV-associated oropharyngeal cancer. J. Clin. Oncol. 38, 1050-1058 (2020)

190. Holsinger, F. C. \& Ferris, R. L. Transoral endoscopic head and neck surgery and its role within the multidisciplinary treatment paradigm of oropharynx cancer: robotics, lasers, and clinical trials. J. Clin. Oncol. 33, 3285-3292 (2015)

191. Sinha, P., Haughey, B. H., Kallogjeri, D. \& Jackson, R. S. Long-term analysis of transorally resected $\mathrm{p} 16+$ oropharynx cancer: outcomes and prognostic factors. Laryngoscope 129, 1141-1149 (2019).

192. Mahmoud, O., Sung, K., Civantos, F. J., Thomas, G. R. \& Samuels, M. A. Transoral robotic surgery for oropharyngeal squamous cell carcinoma in the era of human papillomavirus. Head Neck 40, 710-721 (2018).

193. Jackson, R. S. et al. Transoral resection of human papillomavirus (HPV)-positive squamous cell carcinoma of the oropharynx: outcomes with and without adjuvant therapy. Ann. Surg. Oncol. 24, 3494-3501 (2017)

194. Carey, R. M. et al. Increased rate of recurrence and high rate of salvage in patients with human papillomavirus-associated oropharyngeal squamous cell carcinoma with adverse features treated with primary surgery without recommended adjuvant therapy. Head Neck 43, 1128-1141 (2021).

195. Sethia, R. et al. Quality of life outcomes of transoral robotic surgery with or without adjuvant therapy for oropharyngeal cancer. Laryngoscope 128, 403-411 (2018).

196. Ma, D. J. et al. Phase Il evaluation of aggressive dose de-escalation for adjuvant chemoradiotherapy in human papillomavirus-associated oropharynx squamous cell carcinoma. J. Clin. Oncol. 37 1909-1918 (2019).

197. Hargreaves, S., Beasley, M., Hurt, C., Jones, T. M. $\&$ Evans, M. Deintensification of adjuvant treatment after transoral surgery in patients with human papillomavirus-positive oropharyngeal cancer: the conception of the PATHOS study and its development. Front Oncol. 9, 936 (2019).

198. Ferris, R. L. et al. Updated report of a phase II randomized trial of transoral surgical resection followed by low-dose or standard postoperative therapy in resectable p16+ locally advanced oropharynx cancer: a trial of the ECOG-ACRIN cancer research group (E3311). J. Clin. Oncol. 39, 6010 (2021).

199. Ferris, R. L. et al. Transoral robotic surgical resection followed by randomization to low- or standard-dose IMRT in resectable p16+ locally advanced oropharynx cancer: a trial of the ECOG-ACRIN Cancer Research Group (E3311). J. Clin. Oncol. 38, 6500 (2020).

200. Ferris, R. L. et al. Phase II randomized trial of transoral surgery and low-dose intensity modulated radiation therapy in resectable $\mathrm{p} 16+$ locally advanced oropharynx cancer: an ECOG-ACRIN Cancer Research Group trial (E3311). J. Clin. Oncol. 40, 138-149 (2022).

201. Chera, B. S. et al. Phase 2 trial of de-intensified chemoradiation therapy for favorable-risk human papillomavirus-associated oropharyngeal squamous cell carcinoma. Int. J. Radiat. Oncol. Biol. Phys. 93 , 976-985 (2015)

202. Chera, B. S et al. Mature results of a prospective study of deintensified chemoradiotherapy for low-risk human papillomavirus-associated oropharyngeal squamous cell carcinoma. Cancer 124, 2347-2354 (2018).

203. Pearlstein, K. A. et al. Quality of life for patients with favorable-risk HPV-associated oropharyngeal cancer after de-intensified chemoradiotherapy. Int. J. Radiat. Oncol. Biol. Phys. 103, 646-653 (2019).

204. Seiwert, T. Y. et al. Optima: a phase II dose and volume de-escalation trial for human papillomaviruspositive oropharyngeal cancer. Ann. Oncol. 30 297-302 (2019)

205. Marur, S. et al. E1308: phase II trial of induction chemotherapy followed by reduced-dose radiation and weekly cetuximab in patients with HPV-associated resectable squamous cell carcinoma of the oropharynx-ECOG-ACRIN cancer research group. J. Clin. Oncol. 35, 490-497 (2017).

206. Hegde, J. V. et al. Functional outcomes after de-escalated chemoradiation therapy for human papillomavirus-positive oropharyngeal cancer: secondary analysis of a phase 2 trial. Int. J. Radiat. Oncol. Biol. Phys. 100, 647-651 (2018).

207. Chen, A. M. et al. Reduced-dose radiotherapy for human papillomavirus-associated squamous-cell carcinoma of the oropharynx: a single-arm, phase 2 study. Lancet Oncol. 18, 803-811 (2017).

208. Yamamoto, Y. et al. Radiotherapy alone as a possible de-intensified treatment for human papillomavirusrelated locally advanced oropharyngeal squamous cell carcinoma. Int. J. Clin. Oncol. 24, 640-648 (2019).

209. Hall, S. F., Griffiths, R. J., O'Sullivan, B. \& Liu, F. F. The addition of chemotherapy to radiotherapy did not reduce the rate of distant metastases in low-risk HPV-related oropharyngeal cancer in a real-world setting. Head Neck 41, 2271-2276 (2019).

210. Yom, S. S. et al. Reduced-dose radiation therapy for HPV-associated oropharyngeal carcinoma (NRG Oncology HN002). J. Clin. Oncol. 39, 956-965 (2021).

211. Sher, D. J. et al. Radiation therapy for oropharyngeal squamous cell carcinoma: executive summary of an ASTRO evidence-based clinical practice guideline. Pract. Radiat. Oncol. 7, 246-253 (2017).

212. Howard, J. et al. Minimally invasive surgery versus radiotherapy/chemoradiotherapy for small-volume primary oropharyngeal carcinoma. Cochrane Database Syst. Rev. 12, CD010963 (2016).

213. Nichols, A. C. et al. Radiotherapy versus transoral robotic surgery and neck dissection for oropharyngeal squamous cell carcinoma (ORATOR): an open-label, phase 2, randomised trial. Lancet Oncol. 20 1349-1359 (2019).

214. Ferris, R. L. et al. A novel surgeon credentialing and quality assurance process using transoral surgery for oropharyngeal cancer in ECOG-ACRIN Cance Research Group trial E3311. Oral. Oncol. 110 104797 (2020)

215. de Almeida, J. R. et al. Oncologic outcomes after transoral robotic surgery: a multi-institutional study. JAMA Otolaryngol. 141, 1043-1051 (2015).

216. Mehanna, H. et al. Radiotherapy plus cisplatin or cetuximab in low-risk human papillomavirus-positive oropharyngeal cancer (De-ESCALaTE HPV): an open-label randomised controlled phase 3 trial. Lancet 393, 51-60 (2019).

217. Gillison, M. L. et al. Radiotherapy plus cetuximab or cisplatin in human papillomavirus-positive oropharyngeal cancer (NRG Oncology RTOG 1016): a randomised, multicentre, non-inferiority trial. Lancet 393, 40-50 (2019).

218. Oosthuizen, J. C. \& Doody, J. De-intensified treatment in human papillomavirus-positive oropharyngeal cancer. Lancet 393, 5-7 (2019).

219. Guo, T. et al. Characterization of functionally active gene fusions in human papillomavirus related oropharyngeal squamous cell carcinoma. Int $J$. Cancer 139, 373-382 (2016)

220. Dunn, L. A. et al. Phase I study of induction chemotherapy with afatinib, ribavirin, and weekly carboplatin and paclitaxel for stage IVA/IVB human papillomavirus-associated oropharyngeal squamous cell cancer. Head. Neck 40, 233-241 (2018).

221. Frazer, I. H. \& Chandra, J. Immunotherapy for HPV associated cancer. Papillomavirus Res. 8, 100176 (2019).

222. Barra, F. et al. Advances in therapeutic vaccines for treating human papillomavirus-related cervical intraepithelial neoplasia. J. Obstet. Gynaecol. Res. 46, 989-1006 (2020)

223. Smalley Rumfield, C., Pellom, S. T., Morillon, Y. M., Schlom, J. \& Jochems, C. Immunomodulation to enhance the efficacy of an HPV therapeutic vaccine. J. Immunother. Cancer https://doi.org/10.1136/ jitc-2020-000612 (2020).

224. Ferris, R. L. et al. Nivolumab for recurrent squamous-cell carcinoma of the head and neck. N. Engl. J. Med. 375, 1856-1867 (2016).

225. Cohen, E. E. W. et al. Pembrolizumab versus methotrexate, docetaxel, or cetuximab for recurrent or metastatic head-and-neck squamous cell carcinoma (KEYNOTE-040): a randomised, open-label, phase 3 study. Lancet 393, 156-167 (2019).

226. Burtness, B. et al. Pembrolizumab alone or with chemotherapy versus cetuximab with chemotherapy for recurrent or metastatic squamous cell carcinoma of the head and neck (KEYNOTE-048): a randomised, open-label, phase 3 study. Lancet 394, 1915-1928 (2019)

227. Xu, Y. et al. Programmed death-1/programmed death-ligand 1-axis blockade in recurrent or metastatic head and neck squamous cell carcinoma stratified by human papillomavirus status: a systematic review and meta-analysis. Front. Immunol. 12, 645170 (2021).

228. Wang, J. et al. HPV-positive status associated with inflamed immune microenvironment and improved response to anti-PD-1 therapy in head and neck squamous cell carcinoma. Sci. Rep. 9, 13404 (2019).

229. Galvis, M. M. et al Immunotherapy improves efficacy and safety of patients with HPV positive and negative head and neck cancer: a systematic review and meta-analysis. Crit. Rev. Oncol. Hematol. 150 102966 (2020).

230. Patel, J. J., Levy, D. A., Nguyen, S. A. Knochelmann, H. M. \& Day, T. A. Impact of PD-L1 expression and human papillomavirus status in anti-PD1/PDL1 immunotherapy for head and neck squamous cell carcinoma - systematic review and meta-analysis. Head Neck 42, 774-786 (2019).

231. Wong D. J et al Abstract CT123. IMvoke010: randomized phase III study of atezolizumab as adjuvant monotherapy after definitive therapy of squamous cell carcinoma of the head and neck. Cancer Res. 79, (Suppl.) 13 (2019).

232. Leidner, R. et al. Neoadjuvant immunoradiotherapy results in high rate of complete pathological response and clinical to pathological downstaging in locally advanced head and neck squamous cell carcinoma. Immunother Cancer 9, e002485 (2021). 
233. Ferris, R. L. et al. Neoadjuvant nivolumab for patients with resectable HPV-positive and HPV-negative squamous cell carcinomas of the head and neck in the CheckMate 358 trial. J. Immunother. Cancer $\mathbf{9}$, e002568 (2021)

234. Ferrarotto, R. et al. Impact of neoadjuvant durvalumab with or without tremelimumab on CD8+ tumor lymphocyte density, safety, and efficacy in patients with oropharynx cancer: $\mathrm{CIAO}$ trial results. Clin. Cancer Res. 26, 3211-3219 (2020).

235. von Witzleben, A., Wang, C., Laban, S., Savelyeva, N. \& Ottensmeier, C. H. HNSCC: tumour antigens and their targeting by immunotherapy. Cells 9, 2103 (2020).

236. Massarelli, E. et al. Combining immune checkpoint blockade and tumor-specific vaccine for patients with incurable human papillomavirus 16-related cancer: a phase 2 clinical trial. JAMA Oncol. 5, 67-73 (2019).

237. Aggarwal, C. et al. Immunotherapy targeting HPV 16/18 generates potent immune responses in HPV-associated head and neck cancer. Clin. Cancer Res. 25, 110-124 (2019).

238. Fakhry, C. et al. Human papillomavirus and overal survival after progression of oropharyngeal squamous cell carcinoma. J. Clin. Oncol. 32, 3365-3373 (2014).

239. Harbison, R. A. et al. The mutational landscape of recurrent versus nonrecurrent human papillomavirusrelated oropharyngeal cancer. JCI insight 3, e99327 (2018).

240. Gleber-netto, F. O. et al. Variations in HPV function are associated with survival in squamous cell carcinoma. JCl Insight 4, e 124762 (2019).

241. King, A. and Broggio, J. Cancer Registration Statistics, England https://www.ons.gov.uk/ peoplepopulationandcommunity/healthandsocialcare/ conditionsanddiseases/bulletins/

cancerregistrationstatisticsengland/final2016 (2016).

242. Surveillance Research Program SEER Incidence Data, 1975-2018. https://seer.cancer.gov/data/ (National Cancer Institute, 2020).

243. Lechner, M. \& Fenton, T. R. The genomics, epigenomics, and transcriptomics of HPV-associated oropharyngeal cancer - understanding the basis of a rapidly evolving disease. Adv. Genet. 93, 1-56 (2016).

244. Machczyński, P., Majchrzak, E., Niewinski, P., Marchlewska, J. \& Golusiński, W. A review of the 8th edition of the AJCC staging system for oropharyngeal cancer according to HPV status. Eur. Arch. Otorhinolaryngol. 277, 2407-2412 (2020).

245. Adelstein, D. J. et al. Role of treatment deintensification in the management of $\mathrm{p} 16+$ oropharyngeal cancer: ASCO provisional clinical opinion. J. Clin. Oncol. 37, 1578-1589 (2019).

246. Posner, M. R. et al. Survival (OS) and progression-free survival (PFS) results after induction chemotherapy (IC) followed by de-escalated chemoradiotherapy (RDCRT) for locally advanced (LA) HPV positive oropharynx cancer (HPVOPC). J. Clin. Oncol. 39 , 6058 (2021).

247. Palma, D. A. et al. A randomized trial of radiotherapy vs. trans-oral surgery for treatment de-escalation in HPV-associated oropharyngeal squamous cell carcinoma (ORATOR2). Int. J. Rad. Oncol. Biophys. 111, 1324-1325 (2021).

248. Miles, B. A et al. De-escalated adjuvant therapy after transoral robotic surgery for human papillomavirus-related oropharyngeal carcinoma: the Sinai robotic surgery (SIRS) trial. Oncologist 26 504-513 (2021).
249. Swisher-McClure, S. et al. A phase 2 trial of Alternative Volumes of Oropharyngeal Irradiation for De-intensification (AVOID): omission of the resected primary tumor bed after transoral robotic surgery for human papilloma virus-related squamous cell carcinoma of the oropharynx. Int. J. Radiat. Oncol. Biol. Phys. 106, 725-732 (2020).

\section{Acknowledgements}

Work on HPV-positive OPSCC in T.R.F's lab has been supported by the Rosetrees Trust (CM229-CD1) and the Paul Southgate Fund. The authors thank A. Jay (Department of Histopathology, University College London Hospital) for the micrographs shown in Fig. 1.

\section{Author contributions}

All authors made a substantial contribution to all aspects of the preparation of this manuscript.

\section{Competing interests}

The authors declare no competing interests.

\section{Peer review information}

Nature Reviews Clinical Oncology thanks T. Dalianis and the other, anonymous, reviewer(s) for their contribution to the peer review of this work.

\section{Publisher's note}

Springer Nature remains neutral with regard to jurisdictional claims in published maps and institutional affiliations.

\section{Supplementary information}

The online version contains supplementary material available at https://doi.org/10.1038/s41571-022-00603-7.

(c) Springer Nature Limited 2022 